\title{
Article
}

\section{Involvement of GPR17 in Neuronal Fibre Outgrowth}

\author{
Max Braune ${ }^{1}$, Nico Scherf ${ }^{2}$, Claudia Heine ${ }^{1}$, Katja Sygnecka ${ }^{1}$, Thanigaimalai Pillaiyar ${ }^{3}{ }^{\oplus}$, Chiara Parravicini ${ }^{4}{ }^{(1)}$, \\ Bernd Heimrich ${ }^{5}$, Maria P. Abbracchio ${ }^{4}$, Christa E. Müller ${ }^{3}$ ib and Heike Franke ${ }^{1, *(1)}$
}

1 Rudolf Boehm Institute of Pharmacology and Toxicology, Medical Faculty, University of Leipzig, Härtelstr. 16-18, 04107 Leipzig, Germany; Max.Braune@medizin.uni-leipzig.de (M.B.); claudia.b.heine@t-online.de (C.H.); k.sygnecka@gmail.com (K.S.)

2 Methods and Development Group Neural Data Analysis and Statistical Computing, Max Planck Institute for Human Cognitive and Brain Sciences, Stephanstraße 1A, 04103 Leipzig, Germany; nscherf@cbs.mbp

3 Department of Pharmaceutical \& Medicinal Chemistry, Pharmaceutical Institute, University of Bonn, An der Immenburg 4, 53121 Bonn, Germany; thanigai.medchem@gmail.com (T.P.); christa.mueller@uni-bonn.de (C.E.M.)

4 Department of Pharmaceutical Sciences, University of Milan, Via Balzaretti 9, 20133 Milan, Italy; chiara.parravicini@gmail.com (C.P.); mariapia.abbracchio@unimi.it (M.P.A.)

5 Department of Neuroanatomy, Institute of Anatomy and Cell Biology, Center for Basics in NeuroModulation, Faculty of Medicine, University of Freiburg, Albertstr. 23, 79104 Freiburg, Germany; bernd.heimrich@zfn.uni-freiburg.de

* Correspondence: Heike.Franke@medizin.uni-leipzig.de; Tel.: +49-(0)341-9724602; Fax: +49-(0)341-9724609

check for updates

Citation: Braune, M.; Scherf, N.; Heine, C.; Sygnecka, K.; Pillaiyar, T.; Parravicini, C.; Heimrich, B.;

Abbracchio, M.P.; Müller, C.E.; Franke, H. Involvement of GPR17 in Neuronal Fibre Outgrowth. Int. J. Mol. Sci. 2021, 22, 11683. https://doi.org/ $10.3390 /$ ijms222111683

Academic Editors: Dmitry Aminin and Peter Illes

Received: 4 October 2021

Accepted: 24 October 2021

Published: 28 October 2021

Publisher's Note: MDPI stays neutral with regard to jurisdictional claims in published maps and institutional affiliations.

Copyright: (C) 2021 by the authors Licensee MDPI, Basel, Switzerland This article is an open access article distributed under the terms and conditions of the Creative Commons Attribution (CC BY) license (https:// creativecommons.org/licenses/by/ $4.0 /)$.

\begin{abstract}
Characterization of new pharmacological targets is a promising approach in research of neurorepair mechanisms. The G protein-coupled receptor 17 (GPR17) has recently been proposed as an interesting pharmacological target, e.g., in neuroregenerative processes. Using the well-established ex vivo model of organotypic slice co-cultures of the mesocortical dopaminergic system (prefrontal cortex (PFC) and substantia nigra/ventral tegmental area (SN/VTA) complex), the influence of GPR17 ligands on neurite outgrowth from SN/VTA to the PFC was investigated. The growthpromoting effects of Montelukast (MTK; GPR17- and cysteinyl-leukotriene receptor antagonist), the glial cell line-derived neurotrophic factor (GDNF) and of two potent, selective GPR17 agonists (PSB-16484 and PSB-16282) were characterized. Treatment with MTK resulted in a significant increase in mean neurite density, comparable with the effects of GDNF. The combination of MTK and GPR17 agonist PSB-16484 significantly inhibited neuronal growth. qPCR studies revealed an MTK-induced elevated mRNA-expression of genes relevant for neuronal growth. Immunofluorescence labelling showed a marked expression of GPR17 on NG2-positive glia. Western blot and RT-qPCR analysis of untreated cultures suggest a time-dependent, injury-induced stimulation of GPR17. In conclusion, MTK was identified as a stimulator of neurite fibre outgrowth, mediating its effects through GPR17, highlighting GPR17 as an interesting therapeutic target in neuronal regeneration.
\end{abstract}

Keywords: G protein-coupled receptor 17 (GPR17); neurite outgrowth; montelukast; NG2; ex vivo organotypic brain slice co-culture; neurodegeneration and neuroregeneration

\section{Introduction}

Globally, the number of patients dying from and affected by neurological disorders has increased substantially between 1990 and 2015 [1]. In particular, incidence and prevalence of traumatic brain injury (TBI) increased from 1990 to 2016 [2], as well as the prevalence of Parkinson's disease [3] and dementia [4]. These disorders are the biggest health challenges of the century, posing a serious threat to social and healthcare systems as well as to the future of the global economy [5]. Therefore, new strategies of treatments are pivotal to minimize patients' disabilities promoting better life quality and to reduce costs for society.

The G protein-coupled receptor 17 (GPR17) has recently been proposed as an interesting pharmacological target in neuroinflammatory and neurodegenerative diseases [6-12]. 
The involvement of GPR17 in other pathophysiological conditions of the brain has been demonstrated, leading to the suggestion that GPR17 acts as a 'sensor of brain damage' [13]. Under normal physiological conditions, GPR17 is almost exclusively expressed in NG2-glia, also known as oligodendrocyte precursor cells (OPC), which give rise to myelin producing oligodendrocytes both during development and throughout adult life [14]. Own previous data indicate that the GPR17 expression was elevated in human brain specimens from neurosurgical and autoptic cases after TBI [15]. Viganò and co-workers showed, that after cerebral damage induced by acute injury or ischemia in mice, GPR17-positive NG2-glia rapidly reacted to the damage, suggesting these cells are a 'reserve pool' of adult progenitors maintained for repair purposes [16]. Subsequent fate mapping studies in the same model of stroke [8] as well as in two different models of demyelination [17] showed that, as a result of injury, GPR17-expressing cells proliferate and markedly accumulate in regions surrounding the lesions, but that only a low percentage of these cells eventually gives rise to mature myelinating oligodendrocytes, due to an unfavourable local inflammatory milieu. These data suggest that GPR17 could be pharmacologically exploited for the benefit of patients with neurological diseases, provided that inflammation is counteracted with appropriate agents.

The pharmacology of GPR17 can be characterized as atypical [18], as GPR17 responds to a diverse set of ligands $[9,19,20]$. The receptor is phylogenetically related to both purinergic P2Y receptors (P2YRs) and cysteinyl-leukotriene (CysLT) receptors [9]. In 2006, it was discovered that GPR17 is activated by the uracil nucleotides UDP, UDP-glucose and UDPgalactose as well as by the cysteinyl-leukotrienes LTC4 and LTD4 [21]. The known P2 ${ }_{12} \mathrm{R}$ antagonists Cangrelor and Ticagrelor and the cysteinyl-leukotriene receptor (CysLT-R) antagonists Montelukast (MTK) and Pranlukast have also been described as antagonists of GPR17 [7,21]. In 2010, Benned-Jensen and Rosenkilde independently confirmed activation of GPR17 by uracil nucleotides [19], while other groups neither found activation of GPR17 by uracil nucleotides or cysteinyl-leukotrienes, nor inhibition of GPR17 by Cangrelor or Ticagrelor $[20,22,23]$. These varying effects are likely due to the level of expression of GPR17 in the different transfected cell lines utilized, and in the difficulty of preserving the native pharmacological features of the receptor in artificial recombinant systems.

It has been demonstrated that in animal models of stroke treatment with Cangrelor, and also with MTK and blockade of GPR17 with antisense technology, led to decreased infarct volumes $[13,21,24,25]$. In rats, treatment with MTK reduced neuroinflammation, elevated hippocampal neurogenesis and improved learning and memory [26]. These effects were mediated by GPR17 as demonstrated by using gene knock down and knock out strategies [26].

Otherwise, in PC12 cells, that natively express GPR17, treatment with UDP-glucose and LTD4 promoted survival and neurite outgrowth [27]. In a neonatal rat model of ischemic periventricular leukomalacia treatment with UDP-glucose improved the thickness of myelin sheaths, motor dysfunction and cognitive functions [28].

Due to its special pharmacology and distinct results, the role of GPR17 in neuroreparative and neuroregenerative mechanisms has not yet been completely elucidated. In particular, it is not clear if and how signalling of GPR17 is involved in repair mechanisms in projection systems after trauma and whether these can be influenced by treatment with ligands of GPR17.

For this reason, the well-established rat ex vivo model of organotypic slice co-cultures of the mesocortical dopaminergic system was chosen culturing slices of the substantia nigra/ventral tegmental area (SN/VTA) and the prefrontal cortex (PFC) closely together allowing neuronal fibre outgrowth, to grow from one region to the other $[29,30]$. Organotypic slice co-cultures largely preserve the tissue architecture of the brain regions that they originate from [31], thereby modelling the in vivo situation closely [32]. While the preparation of the co-cultures causes a disruption of the already established connections, this model also strongly correlates with the development of fibre projections of physiological neuronal circuits, thus making the organotypic co-cultures a model of both development 
and of axonal regrowth after mechanical injury [30]. Concretely, in the present study it was examined (i) whether pharmacological stimulation or inhibition of GPR17 can promote neurite outgrowth; (ii) the dynamics of a small selection of genes involved in growth and differentiation of neurons, myelination and inflammation depending on the treatment with GPR17 antagonists and agonists; (iii) the temporal expression of GPR17 in the co-cultures; and (iv) which cells might be effectors of any observed effects.

\section{Results}

\subsection{Neurite Fibre Outgrowth Modulation}

To elucidate if modulation of the GPR17 has any effects on neurite outgrowth in organotypic dopaminergic co-cultures the well-established neurite fibre quantification method in our lab was used [33] (for schematic illustration see Figure 1).

A $\quad$ B

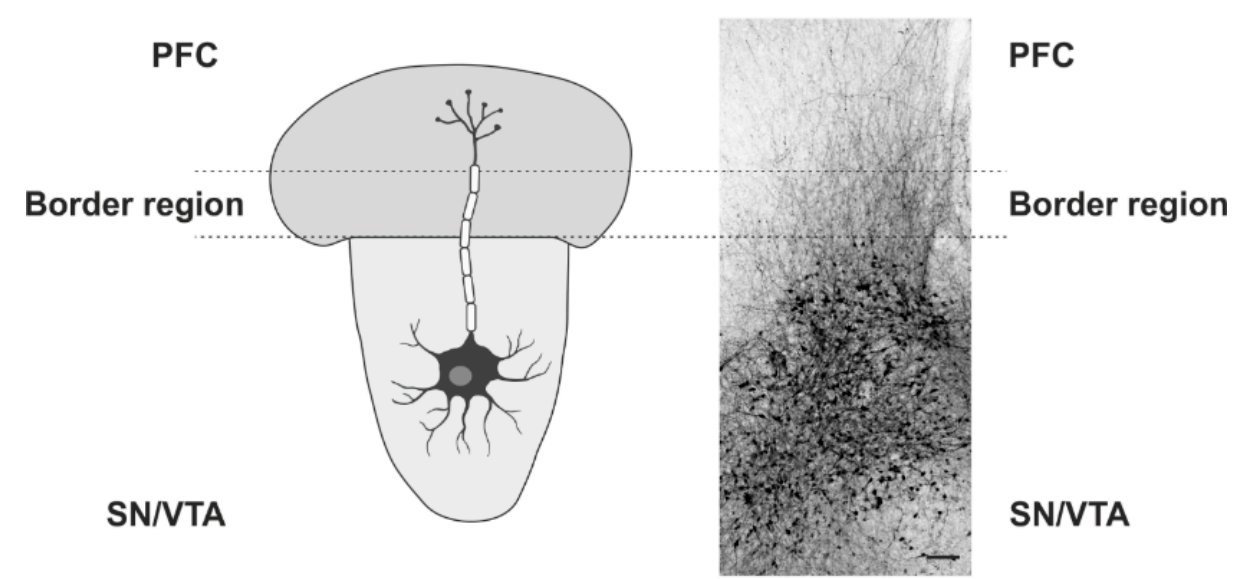

C

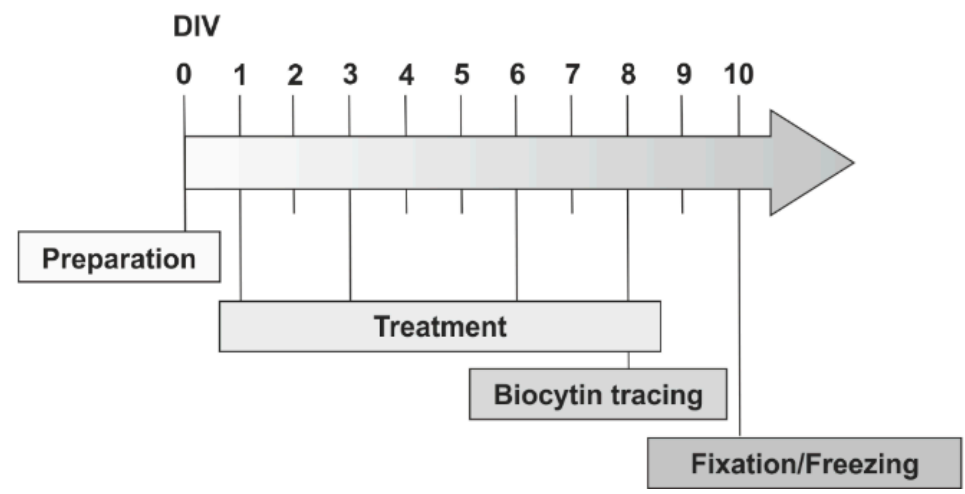

Figure 1. Experimental design. (A) Schematic Illustration of the used organotypic ex vivo slice co-cultures of the dopaminergic system (SN/VTA and PFC) (Attribution: Free neuron vector from vecteezy: Available online: https:/ / www.vecteezy.com/free-vector/nerve-cell (accessed on 23 July 2021). Nerve Cell Vectors by Vecteezy. (B) Overview of the fibre outgrowth visualized by biocytin tracing in an ex vivo co-culture system (rat, fixed at DIV 10). The dotted lines characterize the border region, used for quantification of fibre density. (C) Timeline with the treatment procedures. Scale bar: $\mathbf{B}=200 \mu \mathrm{m}$.

In initial experiments, the effect of Cangrelor (an anti-platelet agent blocking the P2Y ${ }_{12}$ Rs, and reported to act additionally as a non-selective GPR17 antagonist) was investigated, and a significant stimulatory effect on neurite fibre outgrowth in comparison 
to ACSF ( $t$-test with Welch correction $\mathrm{t}(4.34607)=-3.28534 ; p=0.02685$; see Figure S1) was observed.

In the next phase of experiments, different concentrations of MTK, the CysLT1R antagonist and proposed GPR17 antagonist, were employed. In the dopaminergic slice co-cultures, in a similar way to Cangrelor, the application of $10 \mu \mathrm{M}$ of MTK showed a significant stimulation of neurite fibre outgrowth (Figure 2A,C) compared to vehicle (A: $1 \%$ ethanol; C: $0.01 \%$ DMSO, see Section 4.4.) treated controls (one-way ANOVA F $(2,28)=6.143 ; p=0.00614$; followed by Tukey's post hoc test $p=0.0062)$. Neurite fibre outgrowth after application of $1 \mu \mathrm{M}$ MTK was tendentially increased, but not significantly (Tukey's post hoc test $p=0.57299$; Figure 2A).
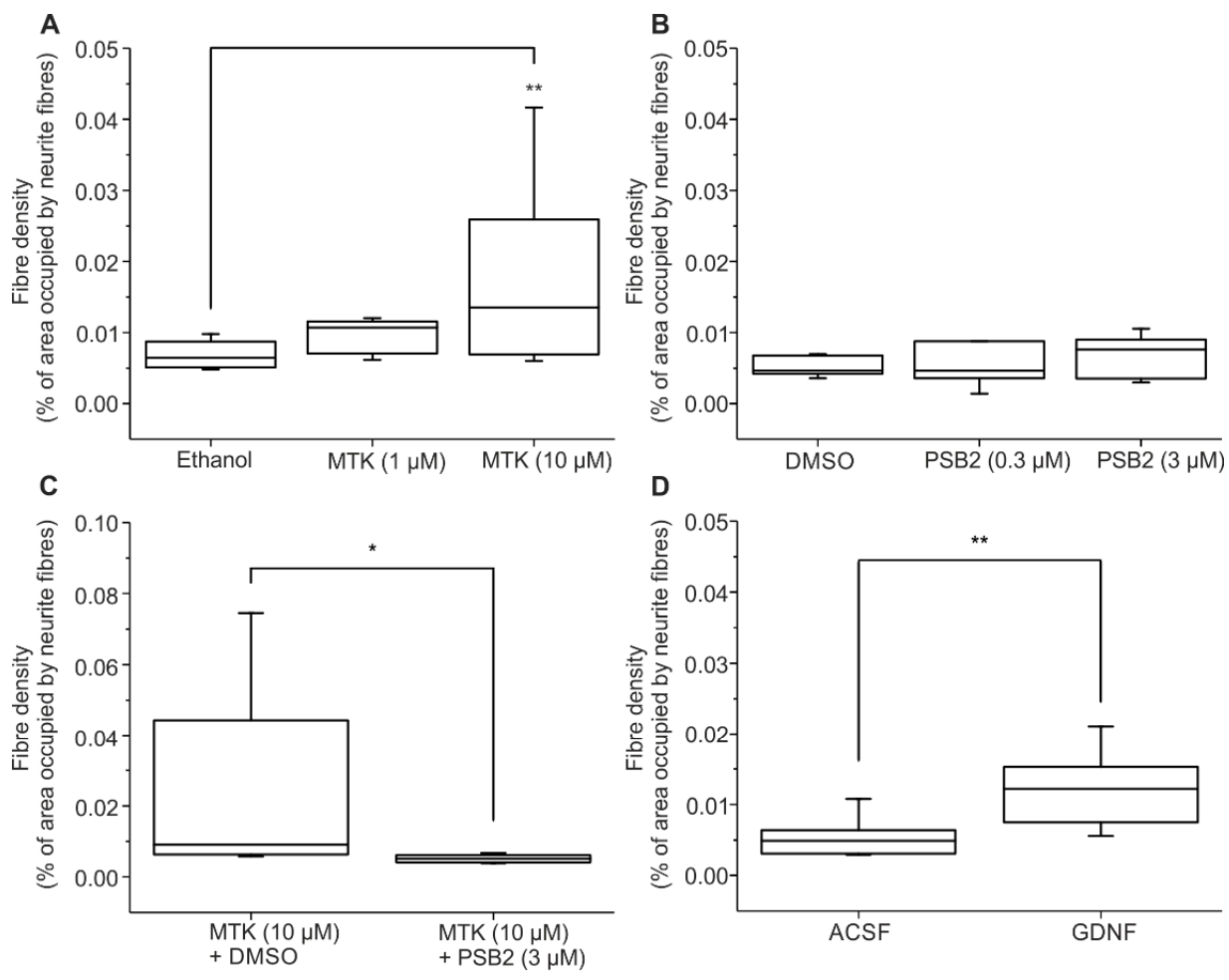

E
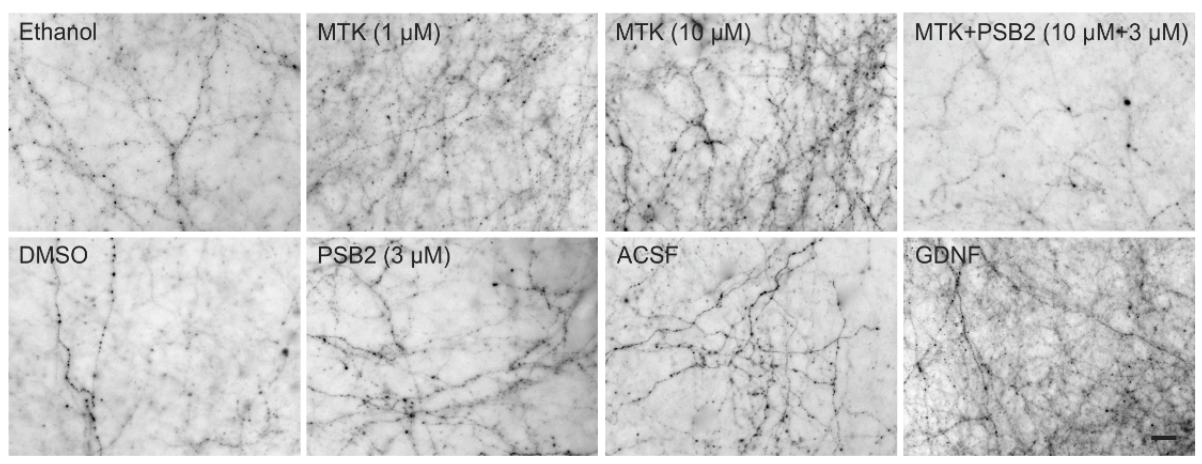

Figure 2. Neurite outgrowth quantification. (A-D) Neurite fibre density was quantified after treatment with (A) MTK, (B) PSB-16484 (PSB2), (C) MTK and PSB-16484 (PSB2) and (D) GDNF using biocytin-tracing. Data are shown as box plots. The number $n$ of animals being used was for (A) $n \geq 9$, (B) $n=4$, (C) $n \geq 5$, (D) $n=8$. For (A,B) ANOVA on Ranks was followed by Tukey's test, for (C) Mann-Whitney test was applied and for (D) $t$-test was applied; ${ }^{*} p<0.05,{ }^{* *} p<0.01$. (E) Pictures of biocytin-labelled fibres in the border region after application of Ethanol, MTK $(1 \mu \mathrm{M})$, MTK $(10 \mu \mathrm{M})$, MTK (10 $\mu \mathrm{M}$, pre-treatment) + PSB-16484 (PSB2, $3 \mu \mathrm{M})$, DMSO, PSB-16484 (PSB2, $3 \mu \mathrm{M})$, ACSF and GDNF. Scale bar: $20 \mu \mathrm{m}$ for all. 
Recently, the small molecule 3-(2-carboxy-4,6-dichloro-indol-3-yl)-propionic acid (MDL29,951) was identified to act as a GPR17 agonist [7,20], and optimized analogues of MDL29,951 (which is non-selective since it also interacts with NMDA receptors), were synthesized $[34,35]$ and characterized in functional assays. In a radioligand binding assay, affinity of this class of compounds for GPR17 was confirmed and blockade by MTK was shown [20,34]. Hence, two of the new, selective GPR17 agonists were used to activate the receptor, namely PSB-16282 and PSB-16484, while MTK was employed as a GPR17 antagonist.

The application of the new GPR17 agonists alone, PSB-16484 (PSB2) (one-way ANOVA $\mathrm{F}(2,19)=0.29054 ; p=0.75113$ ) (Figure 2B) and PSB-16282 (PSB1) (see Figure S2 (one-way ANOVA F $(2,12)=1.04803 ; p=0.38063)$ ), did not cause a significant increase or decrease in neurite fibre outgrowth. Thus, for additional studies, to reduce use of animals only PSB-16484 (PSB2) was utilized.

To evaluate if the observed stimulatory effect of MTK could be antagonized by a new, selective synthetic GPR17 agonist, $10 \mu \mathrm{M}$ MTK was applied in combination with $3 \mu \mathrm{M}$ PSB-16484 (PSB2) and compared to a group treated only with $10 \mu \mathrm{M}$ MTK. Neurite fibre density of cultures treated with MTK and PSB-16484 (PSB2) was significantly lower compared to co-cultures treated only with MTK (Mann-Whitney-Test $\mathrm{U}=28 ; p=0.01732$; Figure 2C) indicating that the stimulation of neurite fibre outgrowth after application of MTK is, at least in part, mediated by GPR17.

As a positive control for stimulation of neurite fibre outgrowth, the well-established neurotrophic factor GDNF, which has been shown to provide neuroprotective and neurotrophic effects especially with regard to the mesencephalon, was used [36]. The application of GDNF ( $50 \mathrm{ng} / \mathrm{mL}$ ) induced a significant stimulation of neurite fibre outgrowth ( $t$-test $\mathrm{t}(14)=-3.34886 ; p=0.00477)$ compared to controls, proving its potency as a neurotrophic factor and demonstrating that neurite fibre outgrowth could be stimulated (Figure 2D).

In comparison, treatment with $10 \mu \mathrm{M}$ MTK showed a 2.6-fold higher mean neurite density compared to controls, while treatment with GDNF led to a 2.3-fold higher mean neurite density compared to controls suggesting that $10 \mu \mathrm{M}$ MTK is a notable stimulator of neurite fibre outgrowth in the organotypic dopaminergic co-culture system. Examples of neurite fibre outgrowth are given in Figure 2E.

\subsection{Gene Expression Analysis of Genes Relevant for Neuronal Growth}

To get further insight into the effects caused by application of MTK and PSB-16484 (PSB2), a gene expression analysis was performed using reverse transcription quantitative real-time polymerase chain reaction (RT-qPCR) after day 10 in culture. In addition to GPR17, the expression of five target genes representative of functions in the central nervous system (CNS) or specific cell populations (Figure 3: PFC (A,C,E,G,I,K); SN/VTA (B,D,F,H,J,L)) (Table 1) were investigated.

GAP43 and NFL were chosen because of their role in axonal regeneration [37] and as regeneration associated genes [38]. In brief, GAP43 is known as being connected to neuronal growth, promoting spontaneous formation of new synapses and enhancing sprouting after injury. Additionally, it is linked to neurite outgrowth, nerve-terminal sprouting and long-term potentiation [39]. Its expression in the used ex vivo slice cocultures has been shown immunohistochemically [30]. In the present study, the expression of mRNA of GAP43 (Figure 3A,B) was significantly elevated in the SN/VTA after treatment with MTK compared to controls (ctrl) (one-way ANOVA F $(2,9)=9.73918 ; p=0.00561$; followed by Tukey's post hoc test $p=0.01067$; Figure 3B) supporting the findings in neurite density quantification. Application of PSB-16484 (PSB2) could not block this effect (Tukey's post hoc test $p=0.99838$ ). There was no significant difference between groups in the PFC (one-way ANOVA F $(2,9)=0.92958 ; p=0.42954$; Figure 3A). 

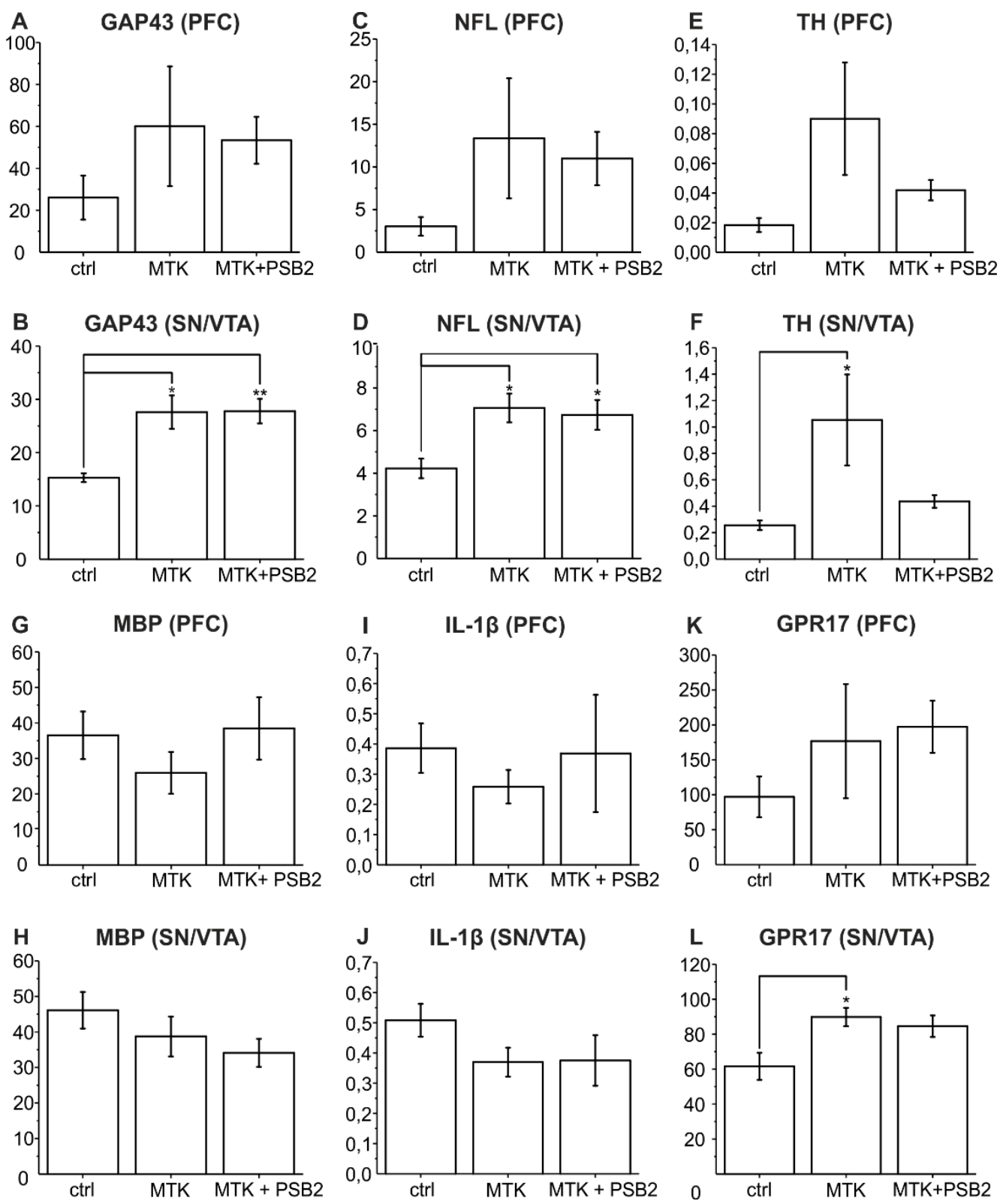

Figure 3. Results of RT-qPCR experiments in the PFC and SN/VTA. Expression of mRNA of GAP43 (A,B), NFL (C,D), TH $(\mathbf{E}, \mathbf{F}), \operatorname{MBP}(\mathbf{G}, \mathbf{H}), \mathrm{IL}-1 \beta(\mathbf{I}, \mathbf{J}), \mathrm{GPR} 17(\mathbf{K}, \mathbf{L})$ is shown on $y$-axis as $\triangle \mathrm{CP}$. X-Axis represents the different treatments $(1 \%$ ethanol and $0.01 \%$ DMSO as controls (ctrl); $10 \mu \mathrm{M}$ MTK and 0.01\% DMSO; $10 \mu \mathrm{M}$ MTK and $3 \mu \mathrm{M}$ PSB-16484 (PSB2)). Statistical analysis was performed in comparison to vehicle control with ANOVA on ranks followed by Tukey's test. Data are shown as bar charts. The number of animals being used was $n=4,{ }^{*} p<0.05,{ }^{* *} p<0.01$.

Table 1. Target genes and primer sequences.

\begin{tabular}{llll}
\hline \multicolumn{1}{c}{ Accession Number } & \multicolumn{1}{c}{ Target } & \multicolumn{1}{c}{ Forward } & \multicolumn{1}{c}{ Reverse } \\
\hline NM_017195.3 & rat GAP43 & ACCACTGATAACTCGCCGTC & TGGCTTCATCTACAGCTTCTTTCT \\
NM_001071777.1 & rat GPR17 & ACTTGTCCTGTGTGCTGGTC & CCCAAAAGGCCAGTGATTGC \\
NM_031512.2 & rat IL-1 $\beta$ & TAGCAGCTTTCGACAGTGAGG & TCTGGACAGCCCAAGTCAAG \\
NM_001025293.1 & rat MBP & TGTGCCACATGTACAAGGACT & TTCATCTTGGGTCCTCTGCG \\
NM_001106116.1 & rat MrpL32 & TTCCGGACCGCTACATAGGTG & CTAGTGCTGGTGCCCACTGAG \\
NM_031783.2 & rat NFL & GCAGCTTACAGGAAACTCTTGG & ACCTGCGAGCTCTGAGAGTA \\
NM_012740.4 & rat TH & TTCTTGAAGGAGCGGACTGG & TGCATTGAAACACGCGGAAG \\
\hline
\end{tabular}


NFL is particularly abundant in axons where it is essential for the radial growth of axons during development, the maintenance of axon calibre and the transmission of electrical impulses [40]. Our results show that mRNA-expression of NFL (Figure 3C,D) was significantly stimulated by treatment with MTK in the SN/VTA (one-way ANOVA $\mathrm{F}(2,9)=6.23136 ; p=0.02002$; followed by Tukey's post hoc test $p=0.02547$; Figure 3D) supporting our findings of higher neurite density in the border region between PFC and SN/VTA after treatment with MTK. The MTK effect was not reduced by PSB-16484 (PSB2) (Tukey's post hoc test $p=0.92805)$. There was no significant difference between groups in the PFC (one-way ANOVA F $(2,9)=1.45291 ; p=0.28391$; Figure 3C).

It should be evaluated, if dopaminergic neurons benefit from application of MTK in the co-culture. Therefore, measurement of the mRNA-expression of $\mathrm{TH}$, the marker enzyme of dopaminergic neurons, was performed [41]. TH mRNA-expression (Figure 3E,F) was significantly elevated in the SN/VTA after treatment with MTK (one-way ANOVA $\mathrm{F}(2,9)=4.29564 ; p=0.04901$; followed by Tukey's post hoc test $p=0.0498)$ and showed non-significantly reduced expression after treatment with PSB-16484 (PSB2) (Tukey's post hoc test $p=0.13171$; Figure 3F). Again, in the PFC there was no significant difference between groups (one-way ANOVA F $(2,9)=2.65474 ; p=0.1241$; Figure 3E).

Additionally, gene expression of myelin basic protein (MBP) was analysed. MBP is an essential part of the myelin sheath insulating axons electrically and thereby allowing saltatory conduction and high conduction velocity [42]. Since GPR17 has been shown to play a role in oligodendrocyte maturation $[43,44]$, it was hypothesized that application of MTK and PSB-16484 (PSB2) could affect expression of the key oligodendrocyte marker MBP. However, expression of mRNA of MBP (Figure 3G,H) showed no significant change after treatment with MTK and PSB-16484 (PSB2) in the PFC (one-way ANOVA F $(2,9)=0.867$; $p=0.45255$; Figure 3G) and the SN/VTA (one-way ANOVA F $(2,9)=1.48997 ; p=0.27609$; Figure $3 \mathrm{H})$.

Furthermore, the question was addressed if the observed neuroregenerative effects of MTK could be assigned to a decreased neuroinflammatory milieu in the dopaminergic co-cultures. Because of its central role in neuroinflammation, IL-1 $\beta$ was chosen as an appropriate marker [45]. However, mRNA-expression of IL-1 $\beta$ (Figure 3I,J) showed no significant changes after treatment with MTK and PSB-16484 (PSB2) but was non-significantly decreased in the PFC (one-way ANOVA F $(2,9)=0.30249 ; p=0.7462$; Figure 3I) and the SN/VTA after treatment with MTK (one-way ANOVA F $(2,9)=0.45195 ; p=0.65008$; Figure 3J). Treatment with PSB-16484 was able to alleviate this tendency in the PFC, but not in the SN/VTA.

Finally, the expression of mRNA of GPR17 (Figure 3K,L) was monitored, which was found to be significantly elevated after treatment with MTK in the SN/VTA (one-way ANOVA F $(2,9)=5.36593$; $p=0.02923$; followed by Tukey's post hoc test $p=0.03199)$, but not in the PFC (one-way ANOVA F $(2,9)=0.94476 ; p=0.42418$; Figure 3K,L). Treatment with PSB-16484 (PSB2) led to no significant difference in expression of GPR17 compared to treatment with MTK in the PFC (Tukey's post hoc test $p=0.96131$ ) and the SN/VTA (Tukey's post hoc test $p=0.83958$ ).

In conclusion, the data show that the mRNA-expression of all three neuronal markers (GAP43, NFL, TH) as well as of GPR17 are significantly increased after treatment with MTK in SN/VTA, but not in the PFC, supporting a neuroregenerative effect after treatment with MTK and suggesting regional differences in responsiveness to MTK.

\subsection{Dynamics of GPR17 Expression in Untreated Organotypic Dopaminergic Co-Cultures}

\subsubsection{RT-qPCR Analysis of GPR17 Expression}

The mRNA expression of GPR17 was characterized at two time points, DIV 3 and DIV 10. In both regions, PFC and SN/VTA, a higher expression was found on DIV 3. The expression of mRNA of GPR17 was significantly reduced after DIV 10 compared to DIV 3 in the PFC ( $t$-test $t(4)=3.22794 ; p=0.03204)$ and tendentially reduced in SN/VTA ( $t$-test with Welch correction $t(2.00119)=0.97405 ; p=0.43272$; Figure 4A,B). This corresponds 
well to the Western blot results, also pointing to an increase in expression of the GPR17 caused by the preparation (injury-induced increase in GPR17 mRNA) (Figure 4C,D).

A
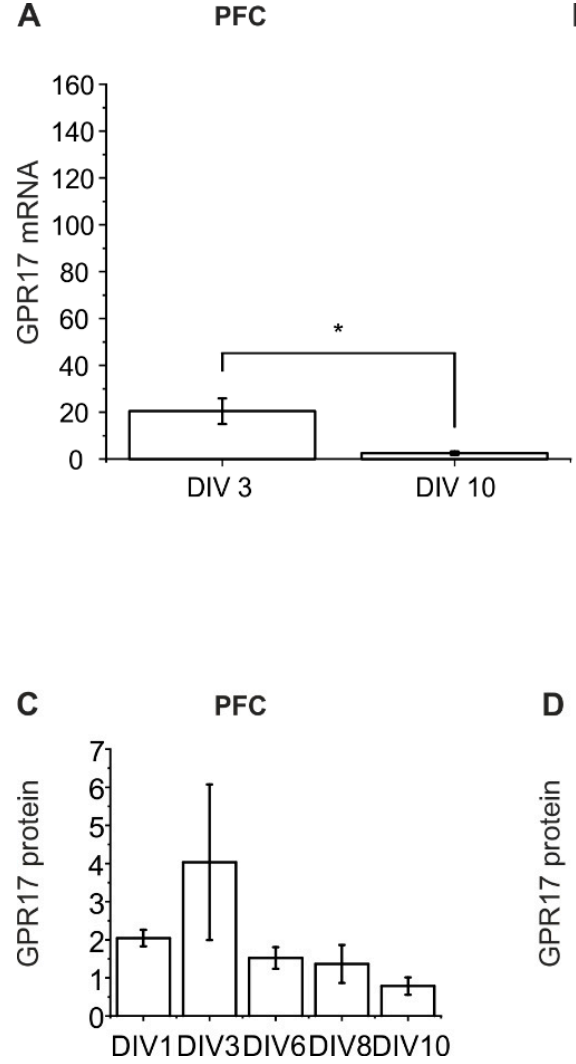

B SN/VTA

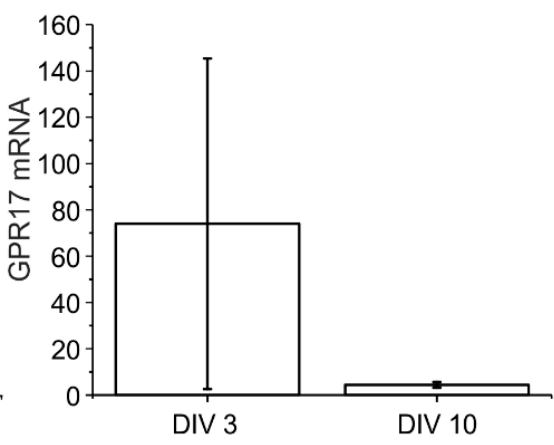

E

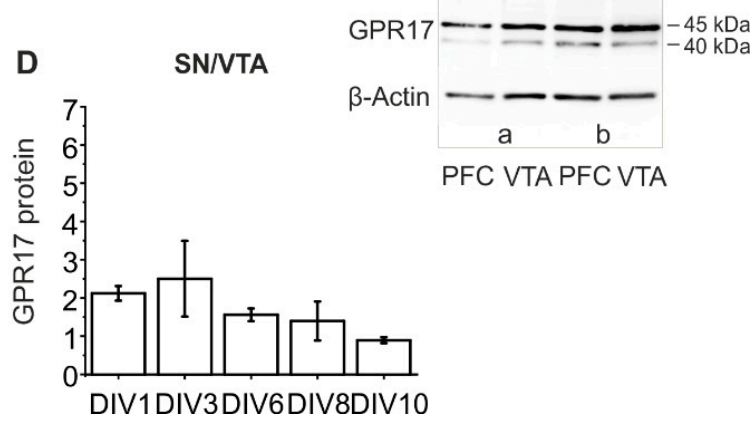

Figure 4. Results of RT-qPCR and Western blot of GPR17 in the ex vivo co-cultures. Expression of mRNA of the GPR17 is shown as $\triangle$ CP in the PFC (A) and SN/VTA (B) after DIV 3 and DIV 10. Statistical analysis was performed in comparison to vehicle control with ANOVA on ranks followed by Tukey's test. Data are shown as bar charts. The number of animals was $n=3,{ }^{*} p<0.05$. Protein expression of GPR17 is shown on DIV 1, 3, 6, 8 and 10 in PFC (C) and SN/VTA (D), data are shown as bar charts. The number of animals being used was $n \geq 3$. For statistical analysis one-way ANOVA (PFC) and Kruskal-Wallis (SN/VTA) was used. (E) Representative examples of Western blot images of samples obtained (DIV 10; (a) ctrl (Ethanol); (b) MTK $10 \mu \mathrm{M}$ ).

\subsubsection{Western Blot Analysis}

Using the rabbit anti-GPR17 antibody (Sigma-Aldrich, St. Louis, MO, USA), two protein bands were labelled, one at $40 \mathrm{kDa}$ and one at $45 \mathrm{kDa}$ (Figure $4 \mathrm{E}$ ). These data are in agreement with previously published literature data suggesting that the band at $40 \mathrm{kDa}$ could be a precursor form carrying high mannose oligosaccharide chains [46]. Western blot analysis showed that GPR17 had its peak of expression both in PFC and SN/VTA after DIV 3, followed by a subsequent decrease (Figure 4C,D). There were no significant differences between DIV in the PFC (one-way ANOVA F (1.69403), $p=0.22724$ ) and the SN/VTA (Kruskal-Wallis ANOVA chi-square $(4)=6.47059 ; p=0.16665)$.

Furthermore, for comparison, native tissue taken from young rats (P2 and P13), from both regions, PFC and SN/VTA, (Figure S3) was investigated. The data showed a slight, but not statistically significant increase in the expression of GPR17 at age P13 compared to P2 in PFC ( $t$-test with Welch correction $t(3.81358)=-0.62477 ; p=0.56755)$ and SN $/ V T A$ $(t$-test $\mathrm{t}(4)=-0.15674 ; p=0.88304)$.

In conclusion, the data suggest an injury-induced GPR17 expression in the studied ex vivo model. Under in vivo conditions no significant changes in GPR17 protein expression between day 2 and day 13 were found (no developmental increase in the investigated 
regions). An injury-induced (slice preparation-induced) increase at DIV 3 was postulated. These data are supported by previous experiments using tissue of the complete co-culture (PFC and SN/VTA), indicating an increase at DIV 3 too (data not shown).

\subsection{Immunohistochemical Analysis of GPR17 Expression}

To investigate which cells express GPR17 in the organotypic dopaminergic co-cultures and could therefore be targeted with ligands of GPR17, the immunolabelling of GPR17 and characteristic cell makers was performed (Table 2).

Table 2. Expression of GPR17 in organotypic slice co-cultures.

\begin{tabular}{cc}
\hline Marker & GPR17-Co-Expression \\
\hline Iba1 & + \\
GFAP & - \\
TH & + \\
NeuN & + \\
BIII-Tubulin & - \\
NFL & +++ \\
NG2 & + \\
O4 & + \\
CNPase & + \\
MBP & +
\end{tabular}

The results show that GPR17 is mainly expressed by NG2-positive glia (Figure 5A-C), but also by neurons (low labelling on NeuN-positive cells; examples are given in Figure 5G,H).

GPR17 is rarely expressed by more developed cells of the oligodendroglial cell lineage (O4-, CNPase-positive cells; an example for $\mathrm{O} 4$ is given in Figure 5D-F) and very low in MBP-positive cells, reflecting the peculiar time dependent expression of this receptor during oligodendrocyte maturation [47]. GPR17-labelled cells were observed rather in the proximity of MBP-positive structures (Figure S4A-C). There is also weak expression on microglia (Iba1-positive cells, Figure S4D-F), but no expression on astrocytes (GFAPpositive cells). Other neuronal markers showed only weak (e.g., ßIII-Tubulin-positive cells) or no (TH-positive, NFL-positive cells) co-localization with GPR17 (examples are given in Figure $5 \mathrm{I}, \mathrm{J}, \mathrm{K}, \mathrm{L}$ ). It is important to note that GPR17-positive cells (stars) could be found more in direct proximity of $\beta \mathrm{III}-\mathrm{Tubulin}-\mathrm{TH}$ - and NFL-positive neurons, but not co-expressed on the TH- or NFL-positive structures. It was hypothesized that GPR17 expression was induced in neurons of our co-cultured slices as a result of the experimental procedure, and reflects a neuronal response to the trauma of the slice preparation.

\subsection{Toxicological Analysis}

To ensure that the applied pharmacological substances had no toxic effects on the organotypic slice co-cultures, the LDH release into incubation medium (IM) was measured. Data showed no significant elevation of LDH activity after treatment with $1 \mu \mathrm{M}$ and $10 \mu \mathrm{M}$ MTK compared to untreated control and vehicle-treated control $(1 \%$ ethanol or $0.01 \%$ DMSO; repeated measurement two-way ANOVA with Greenhouse-Geisser correction $\mathrm{F}(0.29814,0.60849)=1.95985 ; p=0.2293)$ e.g., $1 \%$ ethanol; Figure 6$)$. 

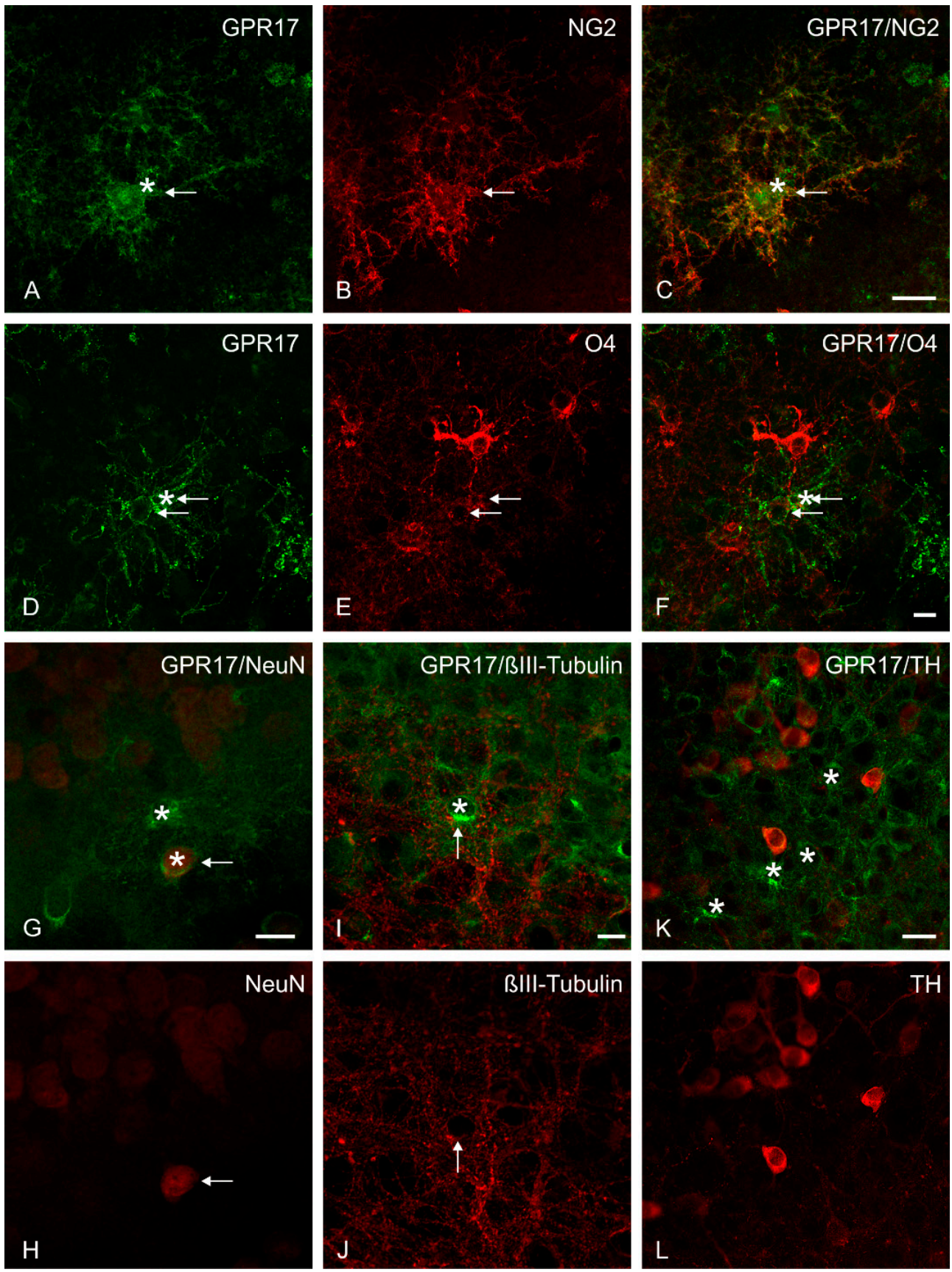

Figure 5. Multiple immunofluorescence study. Representative confocal images of GPR17 expression in organotypic slice co-cultures. At DIV 10 an intense GPR17 immunoreactivity was observed on NG2-positive cells (A-C) and on O4-positive cells (D-F). A low expression of GPR17 (stars) on a small number of cells was observed on $(\mathbf{G}, \mathbf{H})$ NeuN-positive cells and (I,J) $\beta$ III-Tubulin-positive neurons (the thin arrows indicate the co-expression). No co-localization, but a number of GPR17-positive cells (stars) in the proximity were found on $(\mathbf{K}, \mathbf{L})$ TH-positive cells. Scale bars: $(\mathbf{A}-\mathbf{C})=20 \mu \mathrm{m}$; $(\mathbf{D}-\mathbf{J})=10 \mu \mathrm{m} ;(\mathbf{K}, \mathbf{L})=20 \mu \mathrm{m}$. 
A

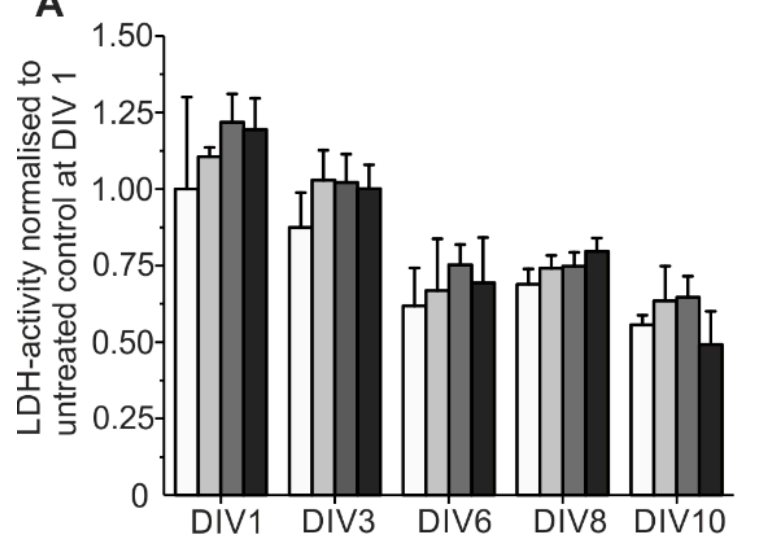

untreated control

vehicle treated control

( $1 \%$ ethanol)

MTK $(1 \mu \mathrm{M})$

MTK $(10 \mu \mathrm{M})$

\section{B}

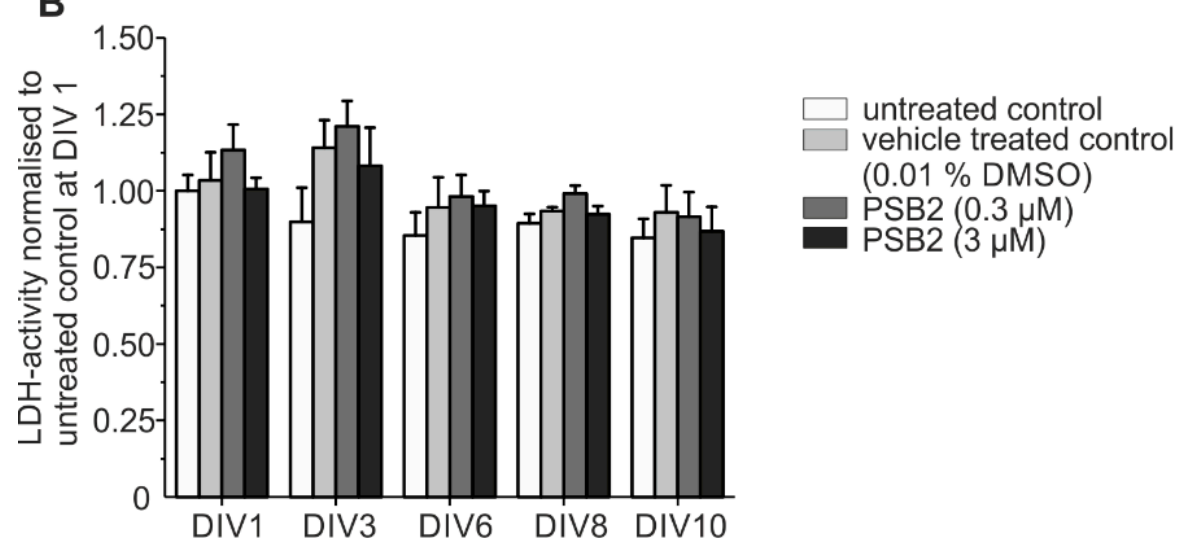

Figure 6. Toxicity testing. (A) LDH activity was measured after treatment with MTK (A) and PSB-16484 (PSB2) (B) compared to vehicle treated control und untreated control. Data have been normalized to the control value at DIV 1 of the respective preparation. Statistical analysis was performed using repeated measurement two-way ANOVA. Each sample represents incubation medium (IM) of one animal. The number of animals was $n=5$.

After treatment with PSB-16484 (PSB2) the LDH activity was neither elevated compared to untreated control nor to vehicle-treated control (repeated measurement two-way ANOVA with Greenhouse-Geisser correction F $(1.6208,6.48319)=4.87266 ; p=0.05565)$ $0.01 \%$ DMSO). Overall LDH activity decreased from DIV 1 to DIV 10 with no significant differences between pharmacological treatment groups and vehicle-treated controls. These data provide proof that neither MTK nor PSB-16484 (PSB2) is toxic to the organotypic dopaminergic slice co-cultures (Figure 6). Both effects, (i) the increase in LDH release (representing the mechanical lesion as a result of the preparation of the co-cultures) and (ii) the decrease in LDH release in the following DIV agrees very well with previous data from our group [48].

\subsection{Electron Microscopy}

The electron microscopic observation revealed the presence of multi-lamellar myelinlike structures in the slice co-cultures of SN/VTA at DIV 10 of incubation (Figure S5A,B) and also in the early postnatal (P12) ventral midbrain (Figure S5C). High resolution images display n-like lamellar organized membranous structures in the SN/VTA part of the coculture. Additionally, in the developing fibres bridge from SN/VTA to the PFC, irregularly organized myelin-like lamellae were found ensheathing neuronal profiles (Figure S5B).

For comparison, in the ventral midbrain $(\mathrm{P} 12$, rat) cross-sectioned axons of varying diameters could be detected. Only very few axons were enveloped by membranous myelin-like sheaths (Figure S5C). 


\section{Discussion}

The present results confirm the ability of MTK to promote axonal outgrowth and suggest the involvement of GPR17 in these effects. In more detail, the obtained data in the dopaminergic organotypic slice co-cultures indicate that (i) MTK, a non-selective GPR17 antagonist, can promote neurite outgrowth with effects comparable to those induced by the well-known neurotrophic factor GDNF, (ii) treatment with MTK increases mRNAexpression of genes relevant to neuronal growth, (iii) a clear expression of GPR17 on NG2glia and in some NeuN-positive neurons, (iv) a time-dependent expression of GPR17 in untreated organotypic dopaminergic co-cultures. These results could be of special interest to patients with TBI and other neurological disorders, suggesting that MTK possibly not only attenuates damage, but also promotes neuroregeneration and repair.

\subsection{Pharmacological Inhibition of the GPR17 with MTK Can Promote Neurite Outgrowth}

The present data show that in the studied dopaminergic organotypic slice co-cultures neuronal outgrowth was stimulated by treatment with the CysLT1-R antagonist and GPR17 antagonist MTK, with effects comparable to those induced by the well-known neurotrophic factor GDNF.

In order to determine if this effect occurred by antagonism of GPR17, two new synthetic GPR17 agonists were applied alone to the co-cultures showing no stimulatory but also no inhibitory effect on neurite outgrowth. Possibly, this reflects the release of endogenous agonists of GPR17 in the slice co-cultures following the preparation procedure, making it difficult to reveal an additional effect caused by the synthetic agonist [49]. Instead, the role of GPR17 was unveiled when the GPR17 agonist PSB-16484 was applied to cultures in the presence of MTK, as shown by abrogation of the MTK induced neurite outgrowth, supporting inhibition of GPR17 as an essential mechanism at the basis of the neuroregenerative effect of MTK under these conditions.

Previous studies in PC12 cells had shown an enhanced neurite outgrowth after treatment with the proposed GPR17 agonist UDP-glucose [27]. While UDP-glucose might have a growth-supporting effect on neurites, its activity as a GPR17 agonist has been questioned $[20,22,23]$. The different outcomes could also be a result of the chosen models investigating neurite outgrowth, as the organotypic slice co-cultures preserve the architecture and microenvironment of the brain with all its cellular players [30] in contrast to the PC12 cell culture model.

Our initial experiments indicated a growth-promoting effect of the employed $\mathrm{P} 2 \mathrm{Y}_{12} \mathrm{R}$ and proposed GPR17 antagonist Cangrelor, which may support a beneficial effect of GPR17 antagonists on neurite outgrowth. However, the effect of Cangrelor (playing a central role in the complex processes of activation and aggregation in blood platelets) [50] may be indirect, involving modulation (inhibition) of inflammatory processes by blocking $\mathrm{P}_{2} \mathrm{Y}_{12} \mathrm{Rs}$ e.g., on microglial cells [50-52].

As the specificity and validity of some ligands of GPR17 have been questioned $[23,53]$ the use of new synthetic ligands of GPR17 is a promising way to unveil its effects. A number of recently published studies support the role of MTK as a GPR17 antagonist. MTK was found to inhibit a synthetic GPR17 agonist $\left[{ }^{3} \mathrm{H}\right] 2$-carboxy-4,6-dichloro- $1 \mathrm{H}$-indole-3propionic acid ( $\left.\left[{ }^{3} \mathrm{H}\right] \mathrm{PSB}-12150\right)$ in a concentration-dependent manner [20]. Furthermore, it was shown that MTK enhanced the growth of neurospheres due to blockade of GPR17 and that knockout of GPR17 led to increased proliferation of neurospheres [26].

\subsection{MTK Promotes Neurite Outgrowth by Elevating Neurotrophic Gene Expression}

In order to gain some insight into the downstream effects following treatment of the slice co-cultures, RT-qPCR was used to investigate genes involved in neuronal growth of dopaminergic neurons, inflammation and myelination.

The presented RT-qPCR results support a growth-promoting effect of MTK in the co-cultures as mRNA-expression of GAP43, NFL and TH is significantly elevated after treatment with MTK in SN/VTA. GAP43 is a regeneration associated gene [38] and over- 
expression of GAP43 has been shown to lead to spontaneous formation of new synapses and enhanced sprouting after injury [39]. By stimulating expression of GAP43, treatment with MTK could promote sprouting of neurites. The neurofilament NFL is essential for the radial growth of axons [40]. Treatment with MTK could thus lead to enhanced axonal growth. TH is known to be the key enzyme of dopaminergic neurons and has been used as the characteristic marker for developing dopaminergic fibres from the SN/VTA to the PFC $[29,41]$. Elevated expression of TH after treatment with MTK underlies MTKs potency to promote growth of dopaminergic neurons in the slice co-cultures.

Therefore, a neurogenic effect of MTK is conceivable as this would also be in line with previously published literature. Transcriptome analysis has shown that GPR17 is specifically highly expressed in adult neural progenitor cells [54]. Recent studies showed that blockade of GPR17 with MTK led to elevated neural stem and progenitor cell proliferation [26,55]. Treatment with MTK resulted in improved cognition of old rats correlating best with enhanced neurogenesis [26]. GPR17 knockdown and knockout in neurospheres induced hyperproliferation and abolished the effects of montelukast [26].

Summarizing, MTK promotes neurite outgrowth in the dopaminergic slice co-culture by enhancing gene expression of neurotrophic genes like GAP43 and NFL. Elevation of mRNA of TH in the SN/VTA after treatment with MTK supports the observed stimulatory effect of neurite fibre outgrowth in the fibre density quantification.

Our data also indicate that GPR17 is not the only mediator of MTKs neurotrophic effects, since MTK induced expression of NFL and GAP43 could not be lowered by treatment with the GPR17 agonist PSB-16484. Only the stimulated mRNA expression of TH was tendentially lowered when co-cultures were treated with PSB-16484, suggesting that elevation of TH could be specifically caused by targeting GPR17 and implying an especially beneficial effect for dopaminergic neurons after blocking GPR17.

\subsection{Is Neurite Outgrowth by MTK Stimulated by Modulation of Neuroinflammatory Pathways?}

The recent literature data from in vitro and in vivo experiments suggests that GPR17 is a sensor of damage [13]. It was shown that MTK reduces neuroinflammation, elevates hippocampal neurogenesis and improves learning and memory in 20-month-old rats [26]. By using gene knockdown and knockout approaches, this effect was demonstrated to be mediated through inhibition of GPR17 reducing microglial activation and elevating neurogenesis [26]. After traumatic brain injury in the human brain an increased expression of GPR17 was found followed by a decrease within a few days [15]. In the present study, in untreated dopaminergic ex vivo co-cultures, expression of GPR17 was high after DIV 3 in RT-qPCR and Western blot results possibly responding to the tissue damage inflicted by the preparation process with upregulation of GPR17. The Western blot data on native (uninjured) tissue (P2, P13) do not show this effect suggesting that GPR17 expression is a time-dependent dynamic parameter in our model possibly responding to the trauma caused by the preparation of the co-cultures and comparable with the in vivo data after TBI.

Inflammatory processes following the preparation procedure (cutting tissue) are conceivable. The authors hypothesized that by treating co-cultures with MTK, a reduction in these neuroinflammatory processes could lead to increased neurite outgrowth. In order to investigate a possible role for neuroinflammatory modulation the mRNA-expression of IL-1 $\beta$ was examined which is known to play a central role in mediating neuroinflammation in pathologies of the CNS [45]. However, in the present study after DIV 10 and treatment with MTK there was only a tendency of reduced expression of mRNA of IL- $1 \beta$ in the dopaminergic slice co-cultures. In the PFC this tendency seemed to be revoked by treatment with PSB-16484 suggesting involvement of GPR17, but differences between the groups were not statistically significant. It cannot be excluded that significant effects on expression of IL-1 $\beta$ measured earlier than on DIV10 might have been missed.

Interestingly, a co-localization of GPR17 with the microglial marker Iba1 was observed. Microglia are known to contribute to neuronal death and neurodegenerative pathologies [56], and microglial inhibition has been shown to be neuroprotective [57]. In a previous 
study, it has furthermore been demonstrated, that GPR17 mediates ischemia-like neuronal injury via microglial activation [58]. Recently, it was shown that chronically activated microglia and signalling of GPR17 inhibit maturation of OPC and myelination in an optic nerve injury model [59].

Thus, microglial expression of GPR17 and recent literature support an anti-inflammatory effect of treatment with GPR17 antagonists. The known activity of MTK as a CysLTR1 antagonist suggests a role for anti-inflammatory effects. However, in the organotypic slice co-culture model, significant effects on mRNA-expression of IL-1 $\beta$ after treatment with MTK reflecting anti-inflammatory activity were not confirmed but might have been missed. Hence, a relevant anti-inflammatory effect of treatment with MTK cannot be excluded. Additionally, the effect of Cangrelor may partly be explained by an inhibitory role in microglial activation via $\mathrm{P} 2 \mathrm{Y}_{12} \mathrm{Rs}$ as described above.

\subsection{Is Neurite Outgrowth Stimulated by Targeting Oligodendrocytes in the Co-Culture?}

Oligodendrocytes produce myelin which allows for saltatory impulse propagation, but they also exert important trophic functions for neurons [60-62]. GPR17 plays an important role in oligodendrocyte differentiation $[43,47]$ and was shown to be a cellintrinsic timer of myelination [6]. Expression of GPR17 is necessary to start oligodendrocyte differentiation, but must then be downregulated to allow terminal cells' maturation and myelination $[18,63,64]$. Recently, it was demonstrated that treatment with MTK in a mouse model of stroke increased expression of MBP, numbers of oligodendrocytes and fibre connectivity [65].

On this basis, the expression of GPR17 in the organotypic slice co-culture model using immunohistochemistry was examined. As expected, expression of GPR17 was most abundant on NG2-glia. NG2-glia are OPCs responsible for the generation of mature oligodendrocytes during development and adulthood [14,47].

There is also evidence for NG2-glia maintaining neuronal functions and survival of neurons through regulation of neuroinflammatory pathways [66] and it was shown that NG2-glia are permissive to neurite outgrowth and stabilize sensory axons [67]. Furthermore, participation of the GPR17 to post-acute reactivity of NG2-glia in different injury paradigms was demonstrated [68]. It has been suggested that treatment of NG2-glia with GPR17 ligands could possibly influence their differentiation potential or activate reparative functions [16].

In other models of neurodegeneration, an abnormal increase in GPR17 has invariably been associated with myelin defects and its pharmacological manipulation succeeded in restoring endogenous remyelination. Furthermore, OPCs (isolated from spinal cord of SOD1 ${ }^{\mathrm{G} 93 \mathrm{~A}}$ mice) display defective differentiation compared to control cells, which is rescued by treatment with the GPR17 antagonist MTK [12]. It is concluded that, as a result of either acute injury (e.g., stroke, trauma or demyelination) or genetic defects (as is the case of SOD1 ${ }^{\mathrm{G} 93 \mathrm{~A}}$ mice), GPR17 is initially induced to promote OPC maturation, but its persistence in cells and inability to undergo down regulation unfavourably affects cell's terminal maturation and myelination.

Under these conditions, pharmacological antagonism of GPR17 with MTK alleviates this maturation block, and enables OPCs to resume differentiation, which would in turn possibly promote neurite outgrowth. However, mRNA expression of MBP was not enhanced after treatment with MTK on DIV 10, suggesting that the observed effects of MTK on neurite outgrowth cannot be attributed to a change in myelination in this model. Overall, in the slice co-cultures the degree of myelination was low, as found in the electron microscopic images at DIV 10. An earlier or later onset of myelination after treatment with GPR17 ligands might have been missed since oligodendroglial differentiation and GPR17 expression are coordinated in a temporally complex manner [43]. A longer period of cultivation and shorter intervals of measurement might show differences of myelination caused by treatment with MTK also contributing to increased neurite outgrowth. 
In conclusion, the anti-asthmatic drug MTK promotes neurite outgrowth in a dopaminergic slice co-culture system. This effect can be antagonized by treatment with a new potent synthetic agonist of GPR17, pointing to GPR17 as an interesting regulator of neuroregenerative processes. Treatment with MTK results in elevated mRNA-expression of genes relevant for neuronal growth and elevated expression of the dopaminergic marker enzyme tyrosine hydroxylase. GPR17 is most abundantly expressed in NG2-positive glia, suggesting that these cell types are predominantly influenced by treatment with GPR17 ligands.

The present data are in line with previously published findings showing neuroprotective effects associated with the blockade of GPR17 in other disease models characterized by abnormal and prolonged GPR17 upregulation [12,13,24,25,65]. Moreover, the present results support that the neuroregenerative effects induced by MTK after brain injury are, in part, mediated by antagonism of GPR17 but also by additional mechanisms elevating expression of neurotrophic genes.

Repurposing of 'old' drugs to treat both common and rare diseases is increasingly becoming an attractive proposition, because it involves the use of de-risked compounds, with potentially lower overall development costs and shorter development timelines [69]. Intriguingly, MTK is a well-known drug that is currently part of the German guidelines for the therapy of asthma [70].

The shown putative neuroregenerative potential and its easy accessibility makes MTK an interesting drug for patients with TBI and other neurological diseases.

The data and discussions show the need for further research and development of new dual- and multi-target drugs [71], new strategies [72] and new relevant models which will help improving therapeutic strategies.

\section{Materials and Methods}

\subsection{Materials}

The following substances and factors were used: artificial cerebrospinal fluid (ACSF, composed of (mM) $126 \mathrm{NaCl} ; 2.5 \mathrm{KCl} ; 1.2 \mathrm{NaH}_{2} \mathrm{PO}_{4} ; 1.3 \mathrm{MgCl}_{2}$ and $2.4 \mathrm{CaCl}_{2}, \mathrm{pH} 7.4$; Hospital Pharmacy, University of Leipzig, Germany), biocytin (Sigma-Aldrich Co., St. Louis, MO, USA), dimethyl sulfoxide (DMSO, Applichem GmbH, Darmstadt, Germany), ethanol (VWR Chemicals, Darmstadt, Germany), recombinant human glial derived neurotrophic factor (GDNF; Millipore, Bedford, MA, USA), Montelukast (MTK, Biomol GmbH, Hamburg, Germany), Cangrelor (The Medicines Company, Parsippany-Troy Hills, NJ, USA).

The new potent and selective GPR17 agonists 3-(2-carboxyethyl)-4-fluoro-6-(5-methylhexyloxy)-1H-indole-2-carboxylic acid (PSB-16282, (PSB1), EC $5012 \mathrm{nM}$ ) and 3-(2-carboxyethyl)-4-fluoro-6-iodo-1H-indole-2-carboxylic acid (PSB-16484; (PSB2), EC $5032.1 \mathrm{nM} \mathrm{[34];}$ were synthesized, purified and analysed at the University of Bonn and provided by Professor Dr. C. E. Müller (Pharmaceutical Institute, Pharmaceutical \& Medicinal Chemistry, University of Bonn, Germany).

\subsection{Animals}

Rat breeding was performed in the animal facility of the Rudolf Boehm Institute, Universität Leipzig (see Institutional Review Board Statement). Rats were housed under standard conditions with free access to food and water and a $12 \mathrm{~h}$ light-dark cycle (lights on from 7:00 a.m.). Neonatal rat pups (WISTAR RjHan, own breed) of postnatal day 1-3 (P1-3) for the preparation of the organotypic slice co-cultures were used.

\subsection{Preparation}

The slice co-cultures were prepared from P1-3 neonatal rat pups and cultured following the "static" culture protocol as described previously [29,48,73]; for schematic illustration see Figure 1A,B). In brief, $300 \mu \mathrm{m}$ coronal sections of mesencephalon and forebrain were cut simultaneously using two vibratomes (Leica VT1200 S; Leica VT1000 S; Nussloch, Germany). After the separation, slices of PFC and SN/VTA were transferred into petri dishes filled with $4{ }^{\circ} \mathrm{C}$ preparation medium (PM; Minimum Essential Medium (MEM), 
Thermo Fisher Scientific Inc., Waltham, MA, USA), adding glutamine ( $50 \mu \mathrm{g} / \mathrm{mL}$; Thermo Fisher Scientific Inc.). The separated slices were then placed side by side as co-cultures (PFC with SN/VTA) on moist translucent membrane inserts $(0.4 \mu \mathrm{m}$, Millicell-CM, Millipore) in a six-well plate. The wells were filled with incubation medium (IM; MEM (50\%), Hank's Balanced Salt Solution (25\%), heat-inactivated horse serum (25\%) (all from Thermo Fisher Scientific Inc.); supplemented with glutamine to a final concentration of $2 \mathrm{mM}$ and $0.044 \%$ sodium bicarbonate (Sigma-Aldrich; $\mathrm{pH}$ adjusted to 7.2 ) and the antibiotic Gentamycin $(50 \mu \mathrm{g} / \mathrm{mL}$, AMRESCO, Solon, $\mathrm{OH}, \mathrm{USA})$. The cultures were stored at $37^{\circ} \mathrm{C}$ in $5 \% \mathrm{CO}_{2}$, and the medium was changed thrice weekly [73].

\subsection{Slice Co-Culture Treatment Procedure}

The slice co-cultures were kept for 3 and 10 days in vitro (DIV; see Figure 1C). For the pharmacological substance treatments, the slice co-cultures were divided into different experimental groups and treated with the respective substances at different concentrations (see below). The pharmacological treatment was conducted four times on DIV 1, 3, 6 and 8 while changing the IM.

The following compound concentrations were used (selected based on their potencies): $100 \mathrm{pM}$ Cangrelor, $1 \mu \mathrm{M}$ and $10 \mu \mathrm{M}$ MTK (solved in 1\% ethanol), 0.1 and $1 \mu \mathrm{M}$ PSB16282 (dissolved in 0.01\% DMSO), 0.3 and $3 \mu \mathrm{M}$ PSB-16484 (dissolved in 0.01\% DMSO). The compounds were applied separately and in combination, to investigate if GPR17 agonists and (proposed) GPR17 antagonist could counteract their effects (to verify receptor inhibition, the antagonist was given first followed by the respective mixture of antagonist and agonist after $15 \mathrm{~min}$ ).

The glial cell-line derived neurotrophic factor (GDNF; $50 \mathrm{ng} / \mathrm{mL}$; solved in 1\% ACSF) was used as a positive control. GDNF is known for its neural growth-promoting properties, especially on dopaminergic neurons [74]. All pharmacological substances were tested in comparison to vehicle-treated control co-cultures.

\subsection{Fixation of the Slice Co-Cultures}

To perform neurite fibre outgrowth quantification after treatment procedure (Figure 1B) and immunofluorescence labelling, co-cultures were fixed for $2 \mathrm{~h}$ in a solution containing $4 \%$ paraformaldehyde (Merck, Darmstadt, Germany), 0.1\% glutaraldehyde (Serva Electrophoresis $\mathrm{GmbH}$, Heidelberg, Germany), and $0.2 \%$ picric acid (Sigma-Aldrich) in $0.1 \mathrm{M}$ phosphate buffer (PB; $\mathrm{pH} 7.4$ ). Afterwards, co-cultures were rinsed intensively with $\mathrm{PB}$. Finally, the sections were vibratome-cut into $50 \mu \mathrm{m}$ horizontal sections.

\subsection{Neurite Fibre Tracing Procedure}

According to the previously described protocol [73], on DIV 8 biocytin crystals were placed on top of SN/VTA, incubating for $2 \mathrm{~h}$ allowing the uptake of biocytin and were washed with IM, subsequently.

Then, cultures were re-incubated with IM containing the pharmacological substances as previously described [29,75]. After $48 \mathrm{~h}$ (on DIV 10) the co-cultures were immersionfixed (see above) and cut into $50 \mu \mathrm{m}$ horizontal sections using the vibratome. The uptake of the anterograde tracer biocytin was labelled using the avidin-biotin complex (1:50, ABCElite Kit, Vector Laboratories, Inc., Burlingame, CA, USA) and the nickel/cobalt intensified 3,3'-diaminobenzidine hydrochloride (DAB; Sigma-Aldrich) was used as a chromogen. All dyed sections were transferred on glass slides, dehydrated in a sequence of increasing ethanol concentrations, and covered with Entellan (Merck, Darmstadt, Germany).

\subsection{Neurite Fibre Density Quantification}

It has been shown that dopaminergic neurons in the mesocortical projection system develop their typical innervation pattern in the organotypic slice co-cultures [29]. For quantifying neurite fibre outgrowth from SN/VTA to PFC previously described protocols have been applied $[33,48,73]$. Slices were used for analysis only if they fulfilled defined 
criteria, e.g., the slice cultures should have maintained their cellular organization, the tracer should be placed correctly on the SN/VTA, there had to be a dense network of labelled cell bodies in the SN/VTA, there had to be no labelled cell bodies in the PFC [75].

Image analysis: Raw images from the border region (where the two initially separated brain slices were attached) were taken in 40-fold magnification with an AxioCam ICc 1 camera (Carl Zeiss Jena, Germany) on a light microscope (Axioskop 50; Zeiss Oberkochen, Germany). For quantifying the fibre density, an automated image analysis according to a previously described technique was used [76].

After pre-processing and image binarization, the area occupied by neurite fibres was analysed. In detail, the number of pixels occupied by neurite fibres was divided by the number of pixels of the whole image, giving the percentage of the area occupied by neurite fibres called the neurite fibre density. In the shown data, one sample corresponds to the average value of the neurite fibre density ( $n=4$ slices) measured from one animal (4-9 samples (animals) were used per substance; for details see the legend of Figure 2).

\subsection{Multiple Immunofluorescence Labelling}

The free-floating slices $(50 \mu \mathrm{m})$ were pre-incubated with a blocking solution $(0.05 \mathrm{M}$ Tris-buffered saline (TBS), $\mathrm{pH} 7.6$ ), supplemented with foetal calf serum (FCS, 5\%) and Triton X-100 (TX-100; 0.3\%). After $30 \mathrm{~min}$ the slices were incubated in a mixture of primary antibodies diluted in the blocking solution for $48 \mathrm{~h}$ at $4{ }^{\circ} \mathrm{C}$. The following primary antibodies were used: goat anti-glial fibrillary acidic protein (GFAP; 1:300; Santa Cruz Biotechnology, Inc., Heidelberg, Germany), mouse anti-GFAP (1:1000; Sigma), rabbit antiGPR17 (1:100; Cayman Chemical, Ann Arbor, Michigan, USA), rabbit anti-GPR17 (1:1000; Sigma-Aldrich), goat anti-ionized calcium binding adaptor molecule 1 (Iba1; 1:100; abcam, Cambridge, UK), mouse anti-microtubule associated protein 2 (MAP2; 1:200; Chemicon International, CA, USA), goat anti-MAP2 (1:100; Santa Cruz), rat anti-myelin basic protein (MBP; 1:200; Millipore), rabbit anti-NG2 chondroitin sulphate proteoglycan (NG2; 1:200; Millipore), mouse anti-neurofilament (160 kD, NFL Medium, 1:400; abcam), mouse anti-neuronal nuclei (NeuN) (1:100; Chemicon International), mouse anti-tyrosine hydroxylase (TH; 1:1000; Chemicon International) and mouse anti- $\beta$ III-Tubulin (1:400; Promega, Fitchburg, WI, USA).

After washing the slices with TBS three times for $5 \mathrm{~min}$, secondary antibodies were applied with blocking solution and incubated for $2 \mathrm{~h}$. For the simultaneous visualization of the different primary antisera a mixture of the following secondary antibodies was used, specific for the appropriate species IgG (rabbit, mouse, goat). Carbocyanine (Cy2(1:400), Cy3- (1:800), Cy5- (1:100)) conjugated IgGs; all Jackson ImmunoResearch, West Grove, PA, USA) diluted in the blocking solution were applied for $2 \mathrm{~h}$ at room temperature. Finally, Hoechst 33342 (Hoe, final concentration $40 \mathrm{mg} / \mathrm{mL}$, Molecular Probes, Leiden, Netherlands) was added for nuclear staining for $5 \mathrm{~min}$ in TBS at room temperature. After intensive washing and mounting on glass slides, sections were dehydrated and covered with Entellan (Merck).

No immunofluorescence was observed when slices were incubated in TBS without the primary antibody.

Image analysis: Multiple immunofluorescence was investigated by using a confocal laser scanning microscope (LSM 510 Meta, Zeiss, Oberkochen, Germany) working with excitation wavelengths of $488 \mathrm{~nm}$ (argon, yellow-green Cy2-immunofluorescence), $543 \mathrm{~nm}$ (helium/neon1, red Cy3-immunofluorescence), and $633 \mathrm{~nm}$ (helium/neon2, blue Cy5immunofluorescence). An ultraviolet laser $(362 \mathrm{~nm})$ was used to excite the blue-cyan Hoe 33342 fluorescence.

\subsection{Analysis of mRNA-Expression}

The tissue of the dopaminergic slice co-cultures was obtained after DIV 10. After putting the membrane inserts in $4{ }^{\circ} \mathrm{C}$ cold phosphate buffer solution (PBS; pH 7.3-7.4) PFC and SN/VTA were separated with a scalpel. Out of each well, four slices of PFC 
and four slices of SN/VTA were put into one tube and considered as one sample (the sample preparation was replicated four times, each using an individual animal). Then, TRIzol reagent (Life Technologies, Gaithersburg, MD, USA) was used to isolate RNA following the manufacturer's protocol. After the first centrifugation, ethanol and GlycoBlue (Life Technologies) were added to the samples for optimal visibility of the pellets. RNA integrity and concentration were analysed with NanoDrop 1000 (NanoDrop Technologies, Wilmington, DE, USA).

The cDNA-synthesis was accomplished using the RevertAid ${ }^{\mathrm{TM}}$ H Minus First Strand cDNA Synthesis Kit (Thermo Fisher Scientific Inc.) and a thermal cycler (MJ Research Inc., St. Bruno, QC, Canada). Subsequently, the samples were diluted 20-fold using distilled and RNAse/DNAse free water. Afterwards, $5 \mu \mathrm{L}$ SYBR Green qPCR Master Mix (2X; Thermo Fisher Scientific Inc.) and $1 \mu \mathrm{L}$ primer dilution (5 $\mu \mathrm{M}$ each) were added to $4 \mu \mathrm{L}$ sample dilution. Then, qPCR was performed using a StepOnePlus ${ }^{\mathrm{TM}}$ Real-Time PCR System (Thermo Fisher Scientific Inc.). As reference housekeeping gene (HKG) mitochondrial ribosomal protein L32 (Mrpl32) was chosen.

The expression of the following target genes was analysed using primer sequences of growth associated protein 43 (GAP43), GPR17, interleukin-1 $\beta$ (IL-1 $\beta$ ), MBP, neurofilament light chain (NFL), TH (Table 1). The following primers were supplied by Eurofins Genomics (Ebersberg, Germany): rat GAP43, rat GPR17, rat MBP, rat MrpL32, rat NFL and rat TH. Rat IL-1 $\beta$ was supplied by Sigma-Aldrich.

The Hot Start Polymerase was activated by a $15 \mathrm{~min}$ pre-incubation at $95^{\circ} \mathrm{C}$, followed by 55 amplification cycles at $95{ }^{\circ} \mathrm{C}$ for $10 \mathrm{~s}, 60^{\circ} \mathrm{C}$ for $10 \mathrm{~s}$ and $72{ }^{\circ} \mathrm{C}$ for $10 \mathrm{~s}$.

A melting curve analysis was performed to verify correct qPCR products. The following appropriate controls have been used: no template control (water) and "reversetranscription-minus control", in order to exclude the presence of genomic DNA in the samples. Quantification of gene expression was performed by the $\triangle \mathrm{CP}$ method with MrpL32 serving as reference housekeeping gene. Expression levels of the respective receptors are expressed as $\triangle \mathrm{CP}$ with the HKG MrpL32. Data are shown as bar charts (each sample $n$ is equal to four slices of either PFC or SN/VTA of the respective animal).

\subsection{Western Blot}

At first, PFC and SN/VTA of cultured or native tissue were transferred into one tube, respectively. Then, $5 \mu \mathrm{L}$ of Triton-X lysis buffer $(\mathrm{NaCl}(120 \mathrm{mM}))$, Tris $(25 \mathrm{mM})$, EDTA (1 mM), TX-100 (1\%), $1 \mathrm{mM}$ phenylmethylsulfonyl fluoride (PMSF; Sigma-Aldrich) and protease inhibitor mix (0.5\%; Sigma-Aldrich) were added to the samples at $\mathrm{pH}$ 7.4. The samples were homogenized, $15 \mathrm{~min}$ incubated on ice and then centrifuged at $5000 \times g$ at $4{ }^{\circ} \mathrm{C}$. The supernatants were collected, and protein determination was performed using Pierce $^{\mathrm{TM}}$ BCA Protein Assay Kit (Thermo Fisher Scientific Inc.). The samples were diluted with sample buffer (1.4-dithiothreitol (DTT) (500 mM), Tris (312.5 mM), glycerol (25\%), sodium dodecyl sulphate (SDS, $10 \%)$, bromophenol blue $(0.005 \%)$, pyronin $\mathrm{Y}(0.005 \%)$ ), $\mathrm{pH} 6.8$.

This solution was cooked for $5 \mathrm{~min}$ at $95{ }^{\circ} \mathrm{C}$. For performing SDS-PAGE MiniPROTEAN Tetra Cells (Bio-Rad Laboratories GmbH, Munich, Germany) were used. At first, the $10 \%$ separating SDS polyacrylamide gel was placed in the mini cells. Before the $5 \%$ polyacrylamide stacking gel followed, $200 \mu \mathrm{L}$ isopropanol were placed on top of the separating gel for $30 \mathrm{~min}$ for an ideal flat surface and were then sluiced down. The electrophoresis cells were filled with $1 x$ Laemmli buffer (10x Laemmli buffer: glycin (1.92 M), Tris $(250 \mathrm{mM})$, SDS (1\%)). The calculated sample volume and $10 \mu \mathrm{L}$ molecular weight marker (ColorPlus ${ }^{\mathrm{TM}}$ Prestained Protein Ladder, Broad Range; New England Biolabs Inc., USA) were pipetted into the notches. The electrophoresis cells were connected to a power source and the gels were run for $2 \mathrm{~h}$ at $50 \mathrm{~V}$ and $400 \mathrm{~mA}$.

After the gel electrophoresis, the separating gels were transferred into cathode buffer. Proteins were then transferred onto a polyvinylidene difluoride (PVDF) membrane (Westran; pore size $0.45 \mu \mathrm{m}$ ). The blotting was performed for $90 \mathrm{~min}$ at $1.75 \mathrm{~mA} / \mathrm{cm}^{2}$. Subse- 
quently, the PVDF membrane was stained with Ponceau S dye (Carl Roth GmbH + Co. KG; Karlsruhe, Germany) in order to make the protein bands visible.

The PVDF membrane was incubated with a blocking solution for $1 \mathrm{~h}(5 \%$ milk powder, Tris-buffered saline with Tween20 (TBST)). As primary antibody the rabbit anti-GPR17 antibody (Sigma-Aldrich; 1:1000) was used, which was normalized to $\beta$-Actin (SigmaAldrich; 1:5000). The primary antibody was applied in a blocking solution ( $5 \%$ milk powder, $0.1 \% \mathrm{NaN}_{3}$ ) and incubated for $24 \mathrm{~h}$ at $4{ }^{\circ} \mathrm{C}$. The secondary antibody was incubated in a blocking solution ( $5 \%$ milk powder, TBST) for $1 \mathrm{~h}$ at room temperature.

A chemiluminescent solution was applied (Super Signal West Femto Maximum Sensitivity Substrate; Thermo Fisher Scientific Inc.) and after $60 \mathrm{~s}$ images of the membrane were taken with a CCD camera (Diana II, Raytest, Isotopenmeßgeräte GmBH, Straubenhardt, Germany) and by using ImageJ (open source, Rasband: https://imagej.nih.gov/ij/; accessed on 8 June 2021).

\subsection{Analysis of Cell Injury}

As described previously [48], for all pharmacological treatments it was tested if they were toxic to the organotypic co-cultures by measuring the release of lactate dehydrogenase (LDH) in the IM. Briefly, the samples of IM were collected at DIV 1, 3, 5, 8 and 10 (before the cultures were fixed) and stored at $-20^{\circ} \mathrm{C}$ for short time. When after thawing the samples reached room temperature LDH activity was measured following the protocol described by [76]. The samples were applied to a 96-well plate format using a filter based micro plate reader device (POLAR ${ }^{\circledR}$ star Omega, BMG LABTECH GmbH, Ortenberg, Germany). Briefly, a sample volume of $40 \mu \mathrm{L}$ was pipetted into the wells followed by $80 \mu \mathrm{L}$ reaction reagent ( $40 \mu \mathrm{L}$ phosphate buffer, $20 \mu \mathrm{L}$ sodium pyruvate $(1.9 \mathrm{mM}$; Sigma-Aldrich), $20 \mu \mathrm{L}$ NADH $(166.67 \mu \mathrm{g} / \mathrm{mL}$; Sigma-Aldrich). Then, LDH activity was calculated by measuring the decrease in absorption of NADH at $340 \mathrm{~nm}$ (every $10 \mathrm{~s}$ for $3 \mathrm{~min}$ ).

To exclude variations due to temperature changes between individual sets of measurements, LDH activity of all samples has been normalized to the control sample (untreated) at DIV 1 of the respective preparation.

\subsection{Electron Microscopy}

Fixation: For electron microscopy, rats (three 12-day-old rats) were transcardially perfused using $0.1 \mathrm{M} \mathrm{PB}$ containing $4 \%$ PFA and $1.5 \%$ glutaraldehyde (high purity, Serva). The tissue slice co-cultures were fixed with $0.1 \mathrm{M}$ phosphate buffer (PB) containing $4 \%$ paraformaldehyde and $0.05 \%$ glutaraldehyde (high purity, Serva).

Fixed brains and co-cultures were further processed for electron microscopic analysis of presence of myelin-like elements. Using a Leica vibratome, horizontal sections $(50 \mu \mathrm{m})$ of the mesencephalon were cut and the region of interest (PFC, SN/VTA) dissected. From the co-cultures of PFC and SN/VTA complex vibratome sections containing the connecting bridge between both tissue parts were performed. Selected sections were osmicated in $0.5 \% \mathrm{OsO}_{4}$ in $0.1 \mathrm{M} \mathrm{PB}$ for $30 \mathrm{~min}$, block-stained with $1 \%$ uranyl acetate, dehydrated and flat-embedded in resin (Durcupan, Fluka, Buchs, Switzerland) on glass slides. Ultrathin sections were cut by an Ultracut (Leica) and collected on single-slot Formvar-coated copper grids. Digital images of myelin-like structures/sheets in the regions of interest were taken by a transmission electron microscope Leo $906 \mathrm{E}$ (Zeiss).

\subsection{Statistics}

Data are presented as mean \pm S.E.M. in this study. All data were tested for normality and homogeneity of variance. The applied statistical tests are specified in the figure legends and the results (e.g., ANOVA on ranks). The probability level of 0.05 or less was considered to reflect a statistically significant difference. Significance is given as ${ }^{*} p<0.05,{ }^{* *} p<0.01$. All quantitative data have been analysed with Origin ${ }^{\circledR}$ statistical analysis program (Origin ${ }^{\circledR}$ 2018b, www.originlab.com/2018b, accessed on 23 July 2021). 
Supplementary Materials: All data are available online at https:/ /www.mdpi.com/article/10.339 0/ijms222111683/s1.

Author Contributions: Conceptualization: H.F., C.H., M.P.A. and C.E.M.; formal analysis: M.B., H.F., C.H., M.P.A. and C.E.M.; investigation: M.B., K.S., B.H., N.S., C.P., T.P. and C.H.; writing-original draft: M.B. and H.F.; writing—review \& editing: all authors; supervision: H.F.; project administration: H.F., M.P.A. and C.E.M.; funding acquisition: M.B., C.H. and M.P.A. All authors have read and agreed to the published version of the manuscript.

Funding: This research was funded by an internal stipend of the Medical Faculty Leipzig to M.B.; by funding from the German Federal Ministry of Education and Research (BMBF, PtJ-Bio, 0315883, 0313909) to C.H. and K.S.; by founding from FISM-Fondazione Italiana Sclerosi Multipla (2017/R/1) to M.P.A. and by founding from the Italian Ministry of University and Research (MUR), PRINProgetti di Ricerca di Interesse Nazionale (M.P.A.; Grant no. 2017NSXP8J) to M.P.A.

Institutional Review Board Statement: The study was conducted according to the guidelines of the Declaration of Helsinki, according to the ARRIVE guidelines and German guidelines for the welfare of experimental animals and were approved by the local authorities (Landesdirektion Leipzig (Regional office Saxony); T21/13; T05/16). All possible efforts were made to replace, reduce and refine animal experiments.

Informed Consent Statement: Not applicable.

Data Availability Statement: Data are contained within the article.

Acknowledgments: The authors thank Katrin Becker, Kathrin Krause (Rudolf Boehm Institute of Pharmacology and Toxicology, Leipzig) and Sigrun Nestle (Dept. Neuroanatomy, Institute of Anatomy and Cell Biology, Faculty of Medicine, Albert-Ludwigs-University, Freiburg) for their skilful technical assistance in animal handling, and in the laboratory. The authors are grateful to Jennifer Ding (DAAD RISE Summer Research Internship, 2013).

Conflicts of Interest: The authors declare no conflict of interest. The founders had no role in the design of the study; in the collection, analysis or interpretation of data; in writing of the manuscript; or in the decision on publish the results.

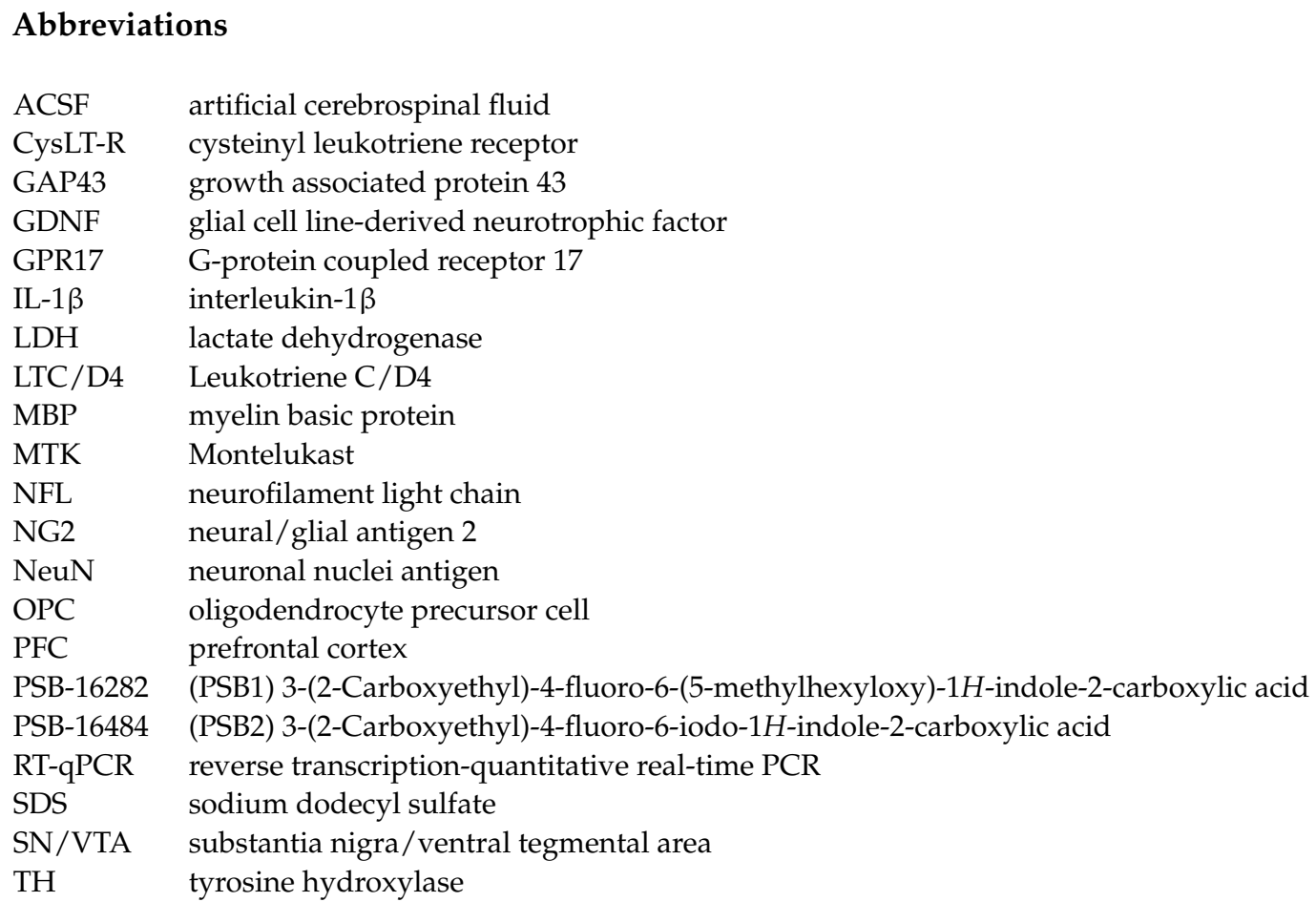




\section{References}

1. Feigin, V.L.; Abajobir, A.A.; Abate, K.H. Global, regional, and national burden of neurological disorders during 1990-2015: A systematic analysis for the global burden of disease study 2015. Lancet Neurol. 2017, 16, 877-897. [CrossRef]

2. James, S.L.; Theadom, A.; Ellenbogen, R.G.; Bannick, M.S.; Montjoy-Venning, W.; Lucchesi, L.R.; Abbasi, N.; Abdulkader, R.; Abraha, H.N.; Adsuar, J.C.; et al. Global, regional, and national burden of traumatic brain injury and spinal cord injury, 1990-2016: A systematic analysis for the global burden of disease study 2016. Lancet Neurol. 2019, 18, 56-87. [CrossRef]

3. Dorsey, E.R.; Elbaz, A.; Nichols, E.; Abd-Allah, F.; Abdelalim, A.; Adsuar, J.C.; Ansha, M.G.; Brayne, C.; Choi, J.-Y.J.; ColladoMateo, D.; et al. Global, regional, and national burden of parkinson's disease, 1990-2016: A systematic analysis for the global burden of disease study 2016. Lancet Neurol. 2018, 17, 939-953. [CrossRef]

4. Nichols, E.; Szoeke, C.E.I.; Vollset, S.E.; Abbasi, N.; Abd-Allah, F.; Abdela, J.; Aichour, M.T.E.; Akinyemi, R.O.; Alahdab, F.; Asgedom, S.W.; et al. Global, regional, and national burden of alzheimer's disease and other dementias, 1990-2016: A systematic analysis for the global burden of disease study 2016. Lancet Neurol. 2019, 18, 88-106. [CrossRef]

5. Olesen, J.; Gustavsson, A.; Svensson, M.; Wittchen, H.U.; Jönsson, B. The economic cost of brain disorders in europe. Eur. J. Neurol. 2012, 19, 155-162. [CrossRef]

6. Chen, Y.; Wu, H.; Wang, S.; Koito, H.; Li, J.; Ye, F.; Hoang, J.; Escobar, S.S.; Gow, A.; Arnett, H.A.; et al. The oligodendrocyte-specific g protein-coupled receptor gpr17 is a cell-intrinsic timer of myelination. Nat. Neurosci. 2009, 12, 1398-1406. [CrossRef] [PubMed]

7. Hennen, S.; Wang, H.; Peters, L.; Merten, N.; Simon, K.; Spinrath, A.; Blättermann, S.; Akkari, R.; Schrage, R.; Schröder, R.; et al. Decoding signaling and function of the orphan $g$ protein-coupled receptor gpr17 with a small-molecule agonist. Sci. Signal. 2013, 6, ra93. [CrossRef] [PubMed]

8. Bonfanti, E.; Gelosa, P.; Fumagalli, M.; Dimou, L.; Viganò, F.; Tremoli, E.; Cimino, M.; Sironi, L.; Abbracchio, M.P. The role of oligodendrocyte precursor cells expressing the gpr17 receptor in brain remodeling after stroke. Cell Death Dis. 2017, 8, e2871. [CrossRef] [PubMed]

9. Fumagalli, M.; Lecca, D.; Coppolino, G.T.; Parravicini, C.; Abbracchio, M.P. Pharmacological properties and biological functions of the gpr17 receptor, a potential target for neuro-regenerative medicine. Adv. Exp. Med. Biol. 2017, 1051, 169-192. [PubMed]

10. Seyedsadr, M.S.; Ineichen, B.V. Gpr17, a player in lysolecithin-induced demyelination, oligodendrocyte survival, and differentiation. J. Neurosci. Off. J. Soc. Neurosci. 2017, 37, 2273-2275. [CrossRef] [PubMed]

11. Alavi, M.S.; Karimi, G.; Roohbakhsh, A. The role of orphan g protein-coupled receptors in the pathophysiology of multiple sclerosis: A review. Life Sci. 2019, 224, 33-40. [CrossRef]

12. Bonfanti, E.; Bonifacino, T.; Raffaele, S.; Milanese, M.; Morgante, E.; Bonanno, G.; Abbracchio, M.P.; Fumagalli, M. Abnormal upregulation of gpr17 receptor contributes to oligodendrocyte dysfunction in sod1 g93a mice. Int. J. Mol. Sci. 2020, 21, 2395. [CrossRef]

13. Lecca, D.; Trincavelli, M.L.; Gelosa, P.; Sironi, L.; Ciana, P.; Fumagalli, M.; Villa, G.; Verderio, C.; Grumelli, C.; Guerrini, U.; et al. The recently identified p2y-like receptor gpr17 is a sensor of brain damage and a new target for brain repair. PLoS ONE 2008, 3, e3579. [CrossRef] [PubMed]

14. Viganò, F.; Dimou, L. The heterogeneous nature of ng2-glia. Brain Res. 2016, 1638, 129-137. [CrossRef] [PubMed]

15. Franke, H.; Parravicini, C.; Lecca, D.; Zanier, E.R.; Heine, C.; Bremicker, K.; Fumagalli, M.; Rosa, P.; Longhi, L.; Stocchetti, N.; et al. Changes of the gpr17 receptor, a new target for neurorepair, in neurons and glial cells in patients with traumatic brain injury. Purinergic Signal. 2013, 9, 451-462. [CrossRef]

16. Viganò, F.; Schneider, S.; Cimino, M.; Bonfanti, E.; Gelosa, P.; Sironi, L.; Abbracchio, M.P.; Dimou, L. Gpr17 expressing ng2-glia: Oligodendrocyte progenitors serving as a reserve pool after injury. Glia 2016, 64, 287-299. [CrossRef]

17. Coppolino, G.T.; Marangon, D.; Negri, C.; Menichetti, G.; Fumagalli, M.; Gelosa, P.; Dimou, L.; Furlan, R.; Lecca, D.; Abbracchio, M.P. Differential local tissue permissiveness influences the final fate of gpr17-expressing oligodendrocyte precursors in two distinct models of demyelination. Glia 2018, 66, 1118-1130. [CrossRef] [PubMed]

18. Lecca, D.; Raffaele, S.; Abbracchio, M.P.; Fumagalli, M. Regulation and signaling of the gpr17 receptor in oligodendroglial cells. Glia 2020, 68, 1957-1967. [CrossRef]

19. Benned-Jensen, T.; Rosenkilde, M.M. Distinct expression and ligand-binding profiles of two constitutively active gpr17 splice variants. Br. J. Pharmacol. 2010, 159, 1092-1105. [CrossRef] [PubMed]

20. Köse, M.; Ritter, K.; Thiemke, K.; Gillard, M.; Kostenis, E.; Müller, C.E. Development of (3)h2-carboxy-4,6-dichloro-1h-indole-3propionic acid ((3)hpsb-12150): A useful tool for studying gpr17. ACS Med. Chem. Lett. 2014, 5, 326-330. [CrossRef]

21. Ciana, P.; Fumagalli, M.; Trincavelli, M.L.; Verderio, C.; Rosa, P.; Lecca, D.; Ferrario, S.; Parravicini, C.; Capra, V.; Gelosa, P.; et al. The orphan receptor gpr17 identified as a new dual uracil nucleotides/cysteinyl-leukotrienes receptor. EMBO J. 2006, 25, 4615-4627. [CrossRef] [PubMed]

22. Qi, A.-D.; Harden, T.K.; Nicholas, R.A. Is gpr17 a p2y/leukotriene receptor? Examination of uracil nucleotides, nucleotide sugars, and cysteinyl leukotrienes as agonists of gpr17. J. Pharmacol. Exp. Ther. 2013, 347, 38-46. [CrossRef] [PubMed]

23. Simon, K.; Merten, N.; Schröder, R.; Hennen, S.; Preis, P.; Schmitt, N.-K.; Peters, L.; Schrage, R.; Vermeiren, C.; Gillard, M.; et al. The orphan receptor gpr17 is unresponsive to uracil nucleotides and cysteinyl leukotrienes. Mol. Pharmacol. 2017, 91, 518-532. [CrossRef] [PubMed] 
24. Ceruti, S.; Viganò, F.; Boda, E.; Ferrario, S.; Magni, G.; Boccazzi, M.; Rosa, P.; Buffo, A.; Abbracchio, M.P. Expression of the new p2y-like receptor gpr17 during oligodendrocyte precursor cell maturation regulates sensitivity to atp-induced death. Glia 2011, 59, 363-378. [CrossRef] [PubMed]

25. Ceruti, S.; Villa, G.; Genovese, T.; Mazzon, E.; Longhi, R.; Rosa, P.; Bramanti, P.; Cuzzocrea, S.; Abbracchio, M.P. The p2y-like receptor gpr17 as a sensor of damage and a new potential target in spinal cord injury. Brain J. Neurol. 2009, 132, $2206-2218$. [CrossRef] [PubMed]

26. Marschallinger, J.; Schäffner, I.; Klein, B.; Gelfert, R.; Rivera, F.J.; Illes, S.; Grassner, L.; Janssen, M.; Rotheneichner, P.; Schmuckermair, C.; et al. Structural and functional rejuvenation of the aged brain by an approved anti-asthmatic drug. Nat. Commun. 2015, 6, 8466. [CrossRef]

27. Daniele, S.; Lecca, D.; Trincavelli, M.L.; Ciampi, O.; Abbracchio, M.P.; Martini, C. Regulation of pc12 cell survival and differentiation by the new p2y-like receptor gpr17. Cell. Signal. 2010, 22, 697-706. [CrossRef]

28. Mao, F.-X.; Li, W.-J.; Chen, H.-J.; Qian, L.-H.; Buzby, J.S. Periventricular leukomalacia long-term prognosis may be improved by treatment with udp-glucose, gdnf, and memantine in neonatal rats. Brain Res. 2012, 1486, 112-120. [CrossRef]

29. Franke, H.; Schelhorn, N.; Illes, P. Dopaminergic neurons develop axonal projections to their target areas in organotypic co-cultures of the ventral mesencephalon and the striatum/prefrontal cortex. Neurochem. Int. 2003, 42, 431-439. [CrossRef]

30. Heine, C.; Sygnecka, K.; Franke, H. Purines in neurite growth and astroglia activation. Neuropharmacology 2016, 104, $255-271$. [CrossRef]

31. Cho, S.; Wood, A.; Bowlby, M.R. Brain slices as models for neurodegenerative disease and screening platforms to identify novel therapeutics. Curr. Neuropharmacol. 2007, 5, 19-33. [CrossRef]

32. Humpel, C. Organotypic brain slice cultures: A review. Neuroscience 2015, 305, 86-98. [CrossRef]

33. Heine, C.; Sygnecka, K.; Scherf, N.; Grohmann, M.; Bräsigk, A.; Franke, H. P2y(1) receptor mediated neuronal fibre outgrowth in organotypic brain slice co-cultures. Neuropharmacology 2015, 93, 252-266. [CrossRef] [PubMed]

34. Baqi, Y.; Pillaiyar, T.; Abdelrahman, A.; Kaufmann, O.; Alshaibani, S.; Rafehi, M.; Ghasimi, S.; Akkari, R.; Ritter, K.; Simon, K.; et al. 3-(2-carboxyethyl)indole-2-carboxylic acid derivatives: Structural requirements and properties of potent agonists of the orphan $\mathrm{g}$ protein-coupled receptor gpr17. J. Med. Chem. 2018, 61, 8136-8154. [CrossRef]

35. Baqi, Y.; Alshaibani, S.; Ritter, K.; Abdelrahman, A.; Spinrath, A.; Kostenis, E.; Müller, C.E. Improved synthesis of 4-/6-substituted 2-carboxy-1h-indole-3-propionic acid derivatives and structure-activity relationships as gpr17 agonists. Med. Chem. Commun. 2014, 5, 86-92. [CrossRef]

36. af Bjerkén, S.; Boger, H.A.; Nelson, M.; Hoffer, B.J.; Granholm, A.-C.; Strömberg, I. Effects of glial cell line-derived neurotrophic factor deletion on ventral mesencephalic organotypic tissue cultures. Brain Res. 2007, 1133, 10-19. [CrossRef]

37. Wang, H.; Wu, M.; Zhan, C.; Ma, E.; Yang, M.; Yang, X.; Li, Y. Neurofilament proteins in axonal regeneration and neurodegenerative diseases. Neural Regen. Res. 2012, 7, 620-626.

38. Ma, T.C.; Willis, D.E. What makes a rag regeneration associated? Front. Mol. Neurosci. 2015, 8, 43. [CrossRef] [PubMed]

39. Benowitz, L.I.; Routtenberg, A. Gap-43: An intrinsic determinant of neuronal development and plasticity. Trends Neurosci. 1997, 20, 84-91. [CrossRef]

40. Yuan, A.; Rao, M.V.; Nixon, R.A. Neurofilaments at a glance. J. Cell Sci. 2012, 125, 3257-3263. [CrossRef] [PubMed]

41. Bademci, G.; Vance, J.M.; Wang, L. Tyrosine hydroxylase gene: Another piece of the genetic puzzle of parkinson's disease. CNS Neurol. Disord. Drug Targets 2012, 11, 469-481. [CrossRef] [PubMed]

42. Fumagalli, M.; Lecca, D.; Abbracchio, M.P. Cns remyelination as a novel reparative approach to neurodegenerative diseases: The roles of purinergic signaling and the p2y-like receptor gpr17. Neuropharmacology 2016, 104, 82-93. [CrossRef]

43. Ou, Z.; Sun, Y.; Lin, L.; You, N.; Liu, X.; Li, H.; Ma, Y.; Cao, L.; Han, Y.; Liu, M.; et al. Olig2-targeted g-protein-coupled receptor gpr17 regulates oligodendrocyte survival in response to lysolecithin-induced demyelination. J. Neurosci. Off. J. Soc. Neurosci. 2016, 36, 10560-10573. [CrossRef] [PubMed]

44. Mendiola, A.S.; Cardona, A.E. The il-1 \textgreek $\{b\}$ phenomena in neuroinflammatory diseases. J. Neural Transm. 2018, 125, 781-795. [CrossRef]

45. Fratangeli, A.; Parmigiani, E.; Fumagalli, M.; Lecca, D.; Benfante, R.; Passafaro, M.; Buffo, A.; Abbracchio, M.P.; Rosa, P. The regulated expression, intracellular trafficking, and membrane recycling of the p2y-like receptor gpr17 in oli-neu oligodendroglial cells. J. Biol. Chem. 2013, 288, 5241-5256. [CrossRef]

46. Fumagalli, M.; Daniele, S.; Lecca, D.; Lee, P.R.; Parravicini, C.; Fields, R.D.; Rosa, P.; Antonucci, F.; Verderio, C.; Trincavelli, M.L.; et al. Phenotypic changes, signaling pathway, and functional correlates of gpr17-expressing neural precursor cells during oligodendrocyte differentiation. J. Biol. Chem. 2011, 286, 10593-10604. [CrossRef] [PubMed]

47. Sygnecka, K.; Heine, C.; Scherf, N.; Fasold, M.; Binder, H.; Scheller, C.; Franke, H. Nimodipine enhances neurite outgrowth in dopaminergic brain slice co-cultures. Int. J. Dev. Neurosci. Off. J. Int. Soc. Dev. Neurosci. 2015, 40, 1-11. [CrossRef]

48. Boccazzi, M.; Lecca, D.; Marangon, D.; Guagnini, F.; Abbracchio, M.P.; Ceruti, S. A new role for the p2y-like gpr17 receptor in the modulation of multipotency of oligodendrocyte precursor cells in vitro. Purinergic Signal. 2016, 12, 661-672. [CrossRef] [PubMed]

49. Gachet, C. P2y(12) receptors in platelets and other hematopoietic and non-hematopoietic cells. Purinergic Signal. 2012, 8, 609-619. [CrossRef] [PubMed]

50. Maeda, M.; Tsuda, M.; Tozaki-Saitoh, H.; Inoue, K.; Kiyama, H. Nerve injury-activated microglia engulf myelinated axons in a p2y12 signaling-dependent manner in the dorsal horn. Glia 2010, 58, 1838-1846. [CrossRef] 
51. Zhan, T.-W.; Tian, Y.-X.; Wang, Q.; Wu, Z.-X.; Zhang, W.-P.; Lu, Y.-B.; Wu, M. Cangrelor alleviates pulmonary fibrosis by inhibiting gpr17-mediated inflammation in mice. Int. Immunopharmacol. 2018, 62, 261-269. [CrossRef]

52. Maekawa, A.; Balestrieri, B.; Austen, K.F.; Kanaoka, Y. Gpr17 is a negative regulator of the cysteinyl leukotriene 1 receptor response to leukotriene d4. Proc. Natl. Acad. Sci. USA 2009, 106, 11685-11690. [CrossRef] [PubMed]

53. Maisel, M.; Herr, A.; Milosevic, J.; Hermann, A.; Habisch, H.-J.; Schwarz, S.; Kirsch, M.; Antoniadis, G.; Brenner, R.; HallmeyerElgner, S.; et al. Transcription profiling of adult and fetal human neuroprogenitors identifies divergent paths to maintain the neuroprogenitor cell state. Stem Cells 2007, 25, 1231-1240. [CrossRef]

54. Huber, C.; Marschallinger, J.; Tempfer, H.; Furtner, T.; Couillard-Despres, S.; Bauer, H.-C.; Rivera, F.J.; Aigner, L. Inhibition of leukotriene receptors boosts neural progenitor proliferation. Cell. Physiol. Biochem. Int. J. Exp. Cell. Physiol. Biochem. Pharmacol. 2011, 28, 793-804. [CrossRef]

55. Kabba, J.A.; Xu, Y.; Christian, H.; Ruan, W.; Chenai, K.; Xiang, Y.; Zhang, L.; Saavedra, J.M.; Pang, T. Microglia: Housekeeper of the central nervous system. Cell. Mol. Neurobiol. 2017, 38, 53-71. [CrossRef] [PubMed]

56. Podbielska, M.; Das, A.; Smith, A.W.; Chauhan, A.; Ray, S.K.; Inoue, J.; Azuma, M.; Nozaki, K.; Hogan, E.L.; Banik, N.L. Neuron-microglia interaction induced bi-directional cytotoxicity associated with calpain activation. J. Neurochem. 2016, 139, 440-455. [CrossRef] [PubMed]

57. Zhao, B.; Wang, H.; Li, C.-X.; Song, S.-W.; Fang, S.-H.; Wei, E.-Q.; Shi, Q.-J. Gpr17 mediates ischemia-like neuronal injury via microglial activation. Int. J. Mol. Med. 2018, 42, 2750-2762. [CrossRef] [PubMed]

58. Wang, J.; He, X.; Meng, H.; Li, Y.; Dmitriev, P.; Tian, F.; Page, J.C.; Lu, Q.R.; He, Z. Robust myelination of regenerated axons induced by combined manipulations of gpr17 and microglia. Neuron 2020, 108, 876-886.e874. [CrossRef]

59. Nave, K.-A. Myelination and support of axonal integrity by glia. Nature 2010, 468, 244-252. [CrossRef] [PubMed]

60. Fünfschilling, U.; Supplie, L.M.; Mahad, D.; Boretius, S.; Saab, A.S.; Edgar, J.; Brinkmann, B.G.; Kassmann, C.M.; Tzvetanova, I.D.; Möbius, W.; et al. Glycolytic oligodendrocytes maintain myelin and long-term axonal integrity. Nature 2012, 485, 517-521. [CrossRef] [PubMed]

61. Lee, Y.; Morrison, B.M.; Li, Y.; Lengacher, S.; Farah, M.H.; Hoffman, P.N.; Liu, Y.; Tsingalia, A.; Jin, L.; Zhang, P.-W.; et al. Oligodendroglia metabolically support axons and contribute to neurodegeneration. Nature 2012, 487, 443-448. [CrossRef]

62. Fumagalli, M.; Bonfanti, E.; Daniele, S.; Zappelli, E.; Lecca, D.; Martini, C.; Trincavelli, M.L.; Abbracchio, M.P. The ubiquitin ligase $\mathrm{mdm} 2$ controls oligodendrocyte maturation by intertwining mtor with g protein-coupled receptor kinase 2 in the regulation of gpr17 receptor desensitization. Glia 2015, 63, 2327-2339. [CrossRef]

63. Simon, K.; Hennen, S.; Merten, N.; Blättermann, S.; Gillard, M.; Kostenis, E.; Gomeza, J. The orphan g protein-coupled receptor gpr17 negatively regulates oligodendrocyte differentiation via $\mathrm{g} \backslash$ textgreek $\{\mathrm{a}\} \mathrm{i} / \mathrm{o}$ and its downstream effector molecules. J. Biol. Chem. 2016, 291, 705-718. [CrossRef] [PubMed]

64. Gelosa, P.; Bonfanti, E.; Castiglioni, L.; Delgado-Garcia, J.M.; Gruart, A.; Fontana, L.; Gotti, M.; Tremoli, E.; Lecca, D.; Fumagalli, M.; et al. Improvement of fiber connectivity and functional recovery after stroke by montelukast, an available and safe anti-asthmatic drug. Pharmacol. Res. 2019, 142, 223-236. [CrossRef] [PubMed]

65. Nakano, M.; Tamura, Y.; Yamato, M.; Kume, S.; Eguchi, A.; Takata, K.; Watanabe, Y.; Kataoka, Y. Ng2 glial cells regulate neuroimmunological responses to maintain neuronal function and survival. Sci. Rep. 2017, 7, 42041. [CrossRef] [PubMed]

66. Busch, S.A.; Horn, K.P.; Cuascut, F.X.; Hawthorne, A.L.; Bai, L.; Miller, R.H.; Silver, J. Adult ng2+ cells are permissive to neurite outgrowth and stabilize sensory axons during macrophage-induced axonal dieback after spinal cord injury. J. Neurosci. Off. J. Soc. Neurosci. 2010, 30, 255-265. [CrossRef]

67. Boda, E.; Viganò, F.; Rosa, P.; Fumagalli, M.; Labat-Gest, V.; Tempia, F.; Abbracchio, M.P.; Dimou, L.; Buffo, A. The gpr17 receptor in ng2 expressing cells: Focus on in vivo cell maturation and participation in acute trauma and chronic damage. Glia 2011, 59, 1958-1973. [CrossRef] [PubMed]

68. Pushpakom, S.; Iorio, F.; Eyers, P.A.; Escott, K.J.; Hopper, S.; Wells, A.; Doig, A.; Guilliams, T.; Latimer, J.; McNamee, C.; et al. Drug repurposing: Progress, challenges and recommendations. Nat. Rev. Drug Discov. 2018, 18, 41-58. [CrossRef] [PubMed]

69. Buhl, R.; Bals, R.; Baur, X.; Berdel, D.; Criée, C.P.; Gappa, M.; Gillissen, A.; Greulich, T.; Haidl, P.; Hamelmann, E.; et al. S2k-leitlinie zur diagnostik und therapie von patienten mit asthma. Pneumologie 2017, 71, 849-919.

70. Pillaiyar, T.; Funke, M.; Al-Hroub, H.; Weyler, S.; Ivanova, S.; Schlegel, J.; Abdelrahman, A.; Müller, C.E. Design, synthesis and biological evaluation of suramin-derived dual antagonists of the proinflammatory g protein-coupled receptors $\mathrm{p} 2 \mathrm{y} 2$ and gpr17. Eur. J. Med. Chem. 2020, 186, 111789. [CrossRef] [PubMed]

71. Parravicini, C.; Lecca, D.; Marangon, D.; Coppolino, G.T.; Daniele, S.; Bonfanti, E.; Fumagalli, M.; Raveglia, L.; Martini, C.; Gianazza, E.; et al. Development of the first in vivo gpr17 ligand through an iterative drug discovery pipeline: A novel disease-modifying strategy for multiple sclerosis. PLoS ONE 2020, 15, e231483. [CrossRef] [PubMed]

72. Heine, C.; Franke, H. Organotypic slice co-culture systems to study axon regeneration in the dopaminergic system ex vivo. Methods Mol. Biol. 2014, 1162, 97-111. [PubMed]

73. Hudson, J.; Granholm, A.C.; Gerhardt, G.A.; Henry, M.A.; Hoffman, A.; Biddle, P.; Leela, N.S.; Mackerlova, L.; Lile, J.D.; Collins, F. Glial cell line-derived neurotrophic factor augments midbrain dopaminergic circuits in vivo. Brain Res. Bull. 1995, 36, 425-432. [CrossRef]

74. Heine, C.; Wegner, A.; Grosche, J.; Allgaier, C.; Illes, P.; Franke, H. P2 receptor expression in the dopaminergic system of the rat brain during development. Neuroscience 2007, 149, 165-181. [CrossRef] 
75. Heine, C.; Sygnecka, K.; Scherf, N.; Berndt, A.; Egerland, U.; Hage, T.; Franke, H. Phosphodiesterase 2 inhibitors promote axonal outgrowth in organotypic slice co-cultures. Neuro-Signals 2013, 21, 197-212. [CrossRef] [PubMed]

76. Koh, J.Y.; Choi, D.W. Quantitative determination of glutamate mediated cortical neuronal injury in cell culture by lactate dehydrogenase efflux assay. J. Neurosci. Methods 1987, 20, 83-90. [CrossRef] 